\title{
Commutative Regular Languages with Product-Form Minimal Automata
}

\author{
Stefan Hoffmann ${ }^{[0000-0002-7866-075 X]}$ \\ Informatikwissenschaften, FB IV, Universität Trier, Universitätsring 15, 54296 Trier, \\ Germany, hoffmanns@informatik.uni-trier.de
}

\begin{abstract}
We introduce a subclass of the commutative regular languages that is characterized by the property that the state set of the minimal deterministic automaton can be written as a certain Cartesian product. This class behaves much better with respect to the state complexity of the shuffle, for which we find the bound $2 \mathrm{~nm}$ if the input languages have state complexities $n$ and $m$, and the upward and downward closure and interior operations, for which we find the bound $n$. In general, only the bounds $(2 \mathrm{~nm})^{|\Sigma|}$ and $n^{|\Sigma|}$ are known for these operations in the commutative case. We prove different characterizations of this class and present results to construct languages from this class. Lastly, in a slightly more general setting of partial commutativity, we introduce other, related, language classes and investigate the relations between them.
\end{abstract}

Keywords: finite automaton $\cdot$ state complexity $\cdot$ shuffle $\cdot$ upward closure $\cdot$ downward closure $\cdot$ commutative language $\cdot$ product-form minimal automaton $\cdot$ partial commutation

\section{Introduction}

The state complexity, as used here, of a regular language $L$ is the minimal number of states needed in a complete deterministic automaton recognizing $L$. The state complexity of an operation on regular languages is the greatest state complexity of the result of this operation as a function of the (maximal) state complexities of its arguments.

Investigating the state complexity of the result of a regularity-preserving operation on regular languages, see [7] for a survey, was first initiated by Maslov in 20] and systematically started by Yu, Zhuang \& Salomaa in 27.

A language is called commutative, if for each word in the language, every permutation of this word is also in the language. The class of commutative automata, which recognize commutative regular languages, was introduced in [2].

The shuffle and iterated shuffle have been introduced and studied to understand the semantics of parallel programs. This was undertaken, as it appears to be, independently by Campbell and Habermann [3, by Mazurkiewicz 22 . and by Shaw 25. They introduced flow expressions, which allow for sequential 
operators (catenation and iterated catenation) as well as for parallel operators (shuffle and iterated shuffle) to specify sequential and parallel execution traces.

The shuffle operation as a binary operation, but not the iterated shuffle, is regularity-preserving on all regular languages. The state complexity of the shuffle operation in the general cases was investigated in [1] for complete deterministic automata and in 4 for incomplete deterministic automata. The bound $2^{n m-1}+$ $2^{(m-1)(n-1)}\left(2^{m-1}-1\right)\left(2^{n-1}-1\right)$ was obtained in the former case, which is not known to be tight, and the tight bound $2^{n m}-1$ in the latter case.

A word is a (scattered) subsequence of another word, if it can be obtained from the latter word by deleting letters. This gives a partial order, and the upward and downward closure and interior operations refer to this partial order. The upward closures are also known as shuffle ideals. The state complexity of these operations was investigated in [11/2 13 1923]

The state complexity of the projection operation was investigated in 171826 . In [26], the tight upper bound $3 \cdot 2^{n-2}-1$ was shown, and in [18] the refined, and tight, bound $2^{n-1}+2^{n-m}-1$ was shown, where $m$ is related to the number of unobservable transitions for the projection operator. Both results were established for incomplete deterministic automata.

In 14 15 16 17 the state complexity of these operations was investigated for commutative regular languages. The results are summarized in Table 1.

\begin{tabular}{|c|c|c|c|}
\hline Operation & Upper Bound & Lower Bound & References \\
\hline$\pi_{\Gamma}(U), \Gamma \subseteq \Sigma$ & $n$ & $n$ & 1417 \\
$U ш V$ & $\min \left\{(2 n m)^{|\Sigma|}, f(n, m)\right\}$ & $\Omega(n m)$ & {$[11415$} \\
$\uparrow U$ & $n^{|\Sigma|}$ & $\Omega\left(\left(\frac{n}{|\Sigma|}\right)^{|\Sigma|}\right)$ & {$[131416]$} \\
$\downarrow U$ & $n^{|\Sigma|}$ & $n$ & $1416]$ \\
$\bigcap U$ & $n^{|\Sigma|}$ & $\Omega\left(\left(\frac{n}{|\Sigma|}\right)^{|\Sigma|}\right)$ & 1416 \\
$\circlearrowleft U$ & $n^{|\Sigma|}$ & $n$ & 1416 \\
$U \cup V, U \cap V$ & $n m$ & tight for each $\Sigma$ & {$[1415$} \\
\hline
\end{tabular}

Table 1. Overview of results for commutative regular languages. The state complexities of the input languages are $n$ and $m$. Also, $f(n, m)=2^{n m-1}+2^{(m-1)(n-1)}\left(2^{m-1}-\right.$ 1) $\left(2^{n-1}-1\right)$ is the general bound for shuffle from [1] in case of complete automata.

\begin{tabular}{|c|c|c|c|}
\hline Operation & Upper Bound & Lower Bound & Reference \\
\hline$\pi_{\Gamma}(U), \Gamma \subseteq \Sigma$ & $n$ & $n$ & Thm. [12 \\
$U \uplus V$ & $2 n m$ & $\Omega(n m)$ & Thm. 112 \\
$\uparrow U, \downarrow U, \Omega U, \circlearrowleft U$ & $n$ & $n$ & Thm. 112 \\
$U \cap V, U \cup V$ & $n m$ & tight for each $\Sigma$ & Thm. 112 \\
\hline
\end{tabular}

Table 2. State Complexity results on the subclass of commutative languages with product-form minimal automaton for input languages with state complexities $n$ and $m$. 
In 8 the minimal commutative automaton was introduced, which can be associated with every commutative regular language. This automaton played a crucial role in 14 15. to derive the bounds mentioned in Table 1. Here, we will investigate the subclass of those language for which the minimal commutative automaton is in fact the smallest automaton recognizing a given commutative language. For this language class, we will derive the following state complexity bounds summarized in Table 2. Additionally, we will prove other characterizations and properties of the subclass considered and relate it with other subclasses, in a more general setting, in the final chapter.

\section{Preliminaries}

In this section and Section [3, we assume that $k \geqslant 0$ denotes our alphabet size and $\Sigma=\left\{a_{1}, \ldots, a_{k}\right\}$ is our alphabet. We will also write $a, b, c$ for $a_{1}, a_{2}, a_{3}$ in case of $|\Sigma| \leqslant 3$. The set $\Sigma^{*}$ denotes the set of all finite sequences over $\Sigma$, i.e., of all words. The finite sequence of length zero, or the empty word, is denoted by $\varepsilon$. For a given word we denote by $|w|$ its length, and for $a \in \Sigma$ by $|w|_{a}$ the number of occurrences of the symbol $a$ in $w$. For $a \in \Sigma$, we set $a^{*}=\{a\}^{*}$. A language is a subset of $\Sigma^{*}$. For $u \in \Sigma^{*}$, the left quotient is $u^{-1} L=\left\{v \in \Sigma^{*} \mid u v \in L\right\}$ and the right quotient is $L u^{-1}=\left\{v \in \Sigma^{*} \mid v u \in L\right\}$.

The shuffle operation, denoted by $\amalg$, is defined by

$$
\begin{aligned}
& u \amalg v=\left\{w \in \Sigma^{*} \mid w=x_{1} y_{1} x_{2} y_{2} \cdots x_{n} y_{n}\right. \text { for some words } \\
& \left.\quad x_{1}, \ldots, x_{n}, y_{1}, \ldots, y_{n} \in \Sigma^{*} \text { such that } u=x_{1} x_{2} \cdots x_{n} \text { and } v=y_{1} y_{2} \cdots y_{n}\right\},
\end{aligned}
$$

for $u, v \in \Sigma^{*}$ and $L_{1} ш L_{2}:=\bigcup_{x \in L_{1}, y \in L_{2}}(x ш y)$ for $L_{1}, L_{2} \subseteq \Sigma^{*}$. If $L_{1}, \ldots, L_{n} \subseteq$ $\Sigma^{*}$, we set $\bigsqcup_{i=1}^{n} L_{i}=L_{1} \amalg \ldots \amalg L_{n}$.

Let $\Gamma \subseteq \Sigma$. The projection homomorphism $\pi_{\Gamma}: \Sigma^{*} \rightarrow \Gamma^{*}$ is given by $\pi_{\Gamma}(x)=x$ for $x \in \Gamma$ and $\pi_{\Gamma}(x)=\varepsilon$ for $x \notin \Gamma$ and extended to $\Sigma^{*}$ by $\pi_{\Gamma}(\varepsilon)=\varepsilon$ and $\pi_{\Gamma}(w x)=\pi_{\Gamma}(w) \pi_{\Gamma}(x)$ for $w \in \Sigma^{*}$ and $x \in \Sigma$. As a shorthand, we set, with respect to a given naming $\Sigma=\left\{a_{1}, \ldots, a_{k}\right\}, \pi_{j}=\pi_{\left\{a_{j}\right\}}$. Then $\pi_{j}(w)=a_{j}^{|w|_{a_{j}}}$.

A language $L \subseteq \Sigma^{*}$ is commutative, if, for $u, v \in \Sigma^{*}$ such that $|v|_{x}=|u|_{x}$ for every $x \in \Sigma$, we have $u \in L$ if and only if $v \in L$, i.e., $L$ is closed under permutation of letters in words from $L$.

A quintuple $\mathcal{A}=\left(\Sigma, Q, \delta, q_{0}, F\right)$ is a finite deterministic and complete automaton (DFA), where $\Sigma$ is the input alphabet, $Q$ the finite set of states, $q_{0} \in Q$ the start state, $F \subseteq Q$ the set of final states and $\delta: Q \times \Sigma \rightarrow Q$ is the totally defined state transition function. Here, we do not consider incomplete automata. The transition function $\delta: Q \times \Sigma \rightarrow Q$ extends to a transition function on words $\delta^{*}: Q \times \Sigma^{*} \rightarrow Q$ by setting $\delta^{*}(q, \varepsilon):=q$ and $\delta^{*}(q, w a):=\delta\left(\delta^{*}(q, w), a\right)$ for $q \in Q, a \in \Sigma$ and $w \in \Sigma^{*}$. In the remainder, we drop the distinction between both functions and also denote this extension by $\delta$. The language recognized by an automaton $\mathcal{A}=\left(\Sigma, Q, \delta, q_{0}, F\right)$ is $L(\mathcal{A})=\left\{w \in \Sigma^{*} \mid \delta\left(q_{0}, w\right) \in F\right\}$. A language $L \subseteq \Sigma^{*}$ is called regular if $L=L(\mathcal{A})$ for some finite automaton $\mathcal{A}$. 
The Nerode right-congruence with respect to $L \subseteq \Sigma^{*}$ is defined, for $u, v \in \Sigma^{*}$, by $u \equiv_{L} v$ if and only if $\forall x \in \Sigma^{*}: u x \in L \Leftrightarrow v x \in L$. The equivalence class of $w \in \Sigma^{*}$ is denoted by $[w]_{\equiv_{L}}=\left\{x \in \Sigma^{*} \mid x \equiv_{L} w\right\}$. A language is regular if and only if the above right-congruence has finite index, and it can be used to define the minimal deterministic automaton $\mathcal{A}_{L}=\left(\Sigma, Q_{L}, \delta_{L},[\varepsilon]_{\equiv_{L}}, F_{L}\right)$ with $Q_{L}=\left\{[u]_{\equiv_{L}} \mid u \in \Sigma^{*}\right\}, \delta_{L}\left([w]_{\equiv_{L}}, a\right)=[w a]_{\equiv_{L}}$ and $F_{L}=\left\{[u]_{\equiv_{L}} \mid u \in L\right\}$. Let $L \subseteq \Sigma^{*}$ be regular with minimal automaton $\mathcal{A}_{L}=\left(\Sigma, Q_{L}, \delta_{L},[\varepsilon]_{\equiv_{L}}, F_{L}\right)$. The number $\left|Q_{L}\right|$ is called the state complexity of $L$ and denoted by $\operatorname{sc}(L)$. The state complexity of a regularity-preserving operation on a class of regular languages is the greatest state complexity of the result of this operation as a function of the (maximal) state complexities for argument languages from the class.

Given two automata $\mathcal{A}=\left(\Sigma, S, \delta, s_{0}, F\right)$ and $\mathcal{B}=\left(\Sigma, T, \mu, t_{0}, E\right)$, an automaton homomorphism $h: S \rightarrow T$ is a map between the state sets such that for each $a \in \Sigma$ and state $s \in S$ we have $h(\delta(s, a))=\mu(h(s), a), h\left(s_{0}\right)=t_{0}$ and $h^{-1}(E)=F$. If $h: S \rightarrow T$ is surjective, then $L(\mathcal{B})=L(\mathcal{A})$. A bijective homomorphism between automata $\mathcal{A}$ and $\mathcal{B}$ is called an isomorphism, and the two automata are said to be isomorphic.

The minimal commutative automaton was introduced in 8 to investigate the learnability of commutative languages. In 1415 this construction was used to define the index and period vector and in the derivation of the state complexity bounds mentioned in Table 1 .

Definition 1 (minimal commutative aut.). Let $L \subseteq \Sigma^{*}$ be regular. The minimal commutative automaton for $L$ is $\mathcal{C}_{L}=\left(\Sigma, S_{1} \times \ldots \times S_{k}, \delta, s_{0}, F\right)$ with

$$
S_{j}=\left\{\left[a_{j}^{m}\right]_{\equiv_{L}}: m \geqslant 0\right\}, \quad F=\left\{\left(\left[\pi_{1}(w)\right]_{\equiv_{L}}, \ldots,\left[\pi_{k}(w)\right]_{\equiv_{L}}\right): w \in L\right\}
$$

and $\delta\left(\left(s_{1}, \ldots, s_{j}, \ldots, s_{k}\right), a_{j}\right)=\left(s_{1}, \ldots, \delta_{j}\left(s_{j}, a_{j}\right), \ldots, s_{k}\right)$ with one-letter transitions $\delta_{j}\left(\left[a_{j}^{m}\right]_{\equiv_{L}}, a_{j}\right)=\left[a_{j}^{m+1}\right]_{\equiv_{L}}$ for $j=1, \ldots, k$ and $s_{0}=\left([\varepsilon]_{\equiv_{L}}, \ldots,[\varepsilon]_{\equiv_{L}}\right)$.

In [8], the next result was shown.

Theorem 2 (Gómez \& Alvarez [8]). Let $L \subseteq \Sigma^{*}$ be a commutative regular language. Then, $L=L\left(\mathcal{C}_{L}\right)$.

In general the minimal commutative automaton is not equal to the minimal deterministic and complete automaton for a regular commutative language $L$, see Example 1.

Example 1. For $L=\left\{\left.w \in \Sigma^{*}|| w\right|_{a}=0\right.$ or $\left.|w|_{b}>0\right\}$ with $\Sigma=\{a, b\}$ the minimal deterministic and complete automaton and the minimal commutative automaton are not the same, see Figure 1. This language is from 8 . In fact, the difference can get quite large, as shown by $L_{p}=\left\{\left.w \in \Sigma^{*}\left|\sum_{j=1}^{k} j \cdot\right| w\right|_{a_{j}} \equiv 0\right.$ $(\bmod p)\}$ for a prime $p>k$. Here, $\operatorname{sc}\left(L_{p}\right)=p$, but $\mathcal{C}_{L_{p}}$ has $p^{k}$ states.

The next definition from [1415] generalizes the notion of a cyclic and noncyclic part for unary automata [24], and the notion of periodic language [6 1415]. 

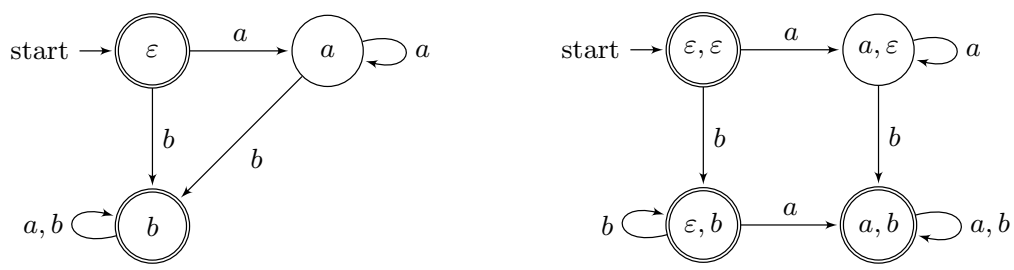

Fig. 1. The minimal deterministic automaton (left) and the minimal commutative automaton (right) of the language $\left\{\left.w \in \Sigma^{*}|| w\right|_{a}=0\right.$ or $\left.|w|_{b}>0\right\}$.

Definition 3 (index and period vector). The index vector $\left(i_{1}, \ldots, i_{k}\right)$ and period vector $\left(p_{1}, \ldots, p_{k}\right)$ for a commutative regular language $L \subseteq \Sigma^{*}$ with minimal commutative automaton $\mathcal{C}_{L}=\left(\Sigma, S_{1} \times \ldots \times S_{k}, \delta, s_{0}, F\right)$ are the unique minimal numbers such that $\delta\left(s_{0}, a_{j}^{i_{j}}\right)=\delta\left(s_{0}, a_{j}^{i_{j}+p_{j}}\right)$ for all $j \in\{1, \ldots, k\}$.

Note that, in Definition 3, we have, for all $j \in\{1, \ldots, k\},\left|S_{j}\right|=i_{j}+p_{j}$. Also note that for unary languages, i.e., if $|\Sigma|=1, \mathcal{C}_{L}$ equals $\mathcal{A}_{L}$ and $i_{1}+p_{1}$ equals the number of states of the minimal automaton.

Example 2. Let $L=(a a)^{*} \uplus(b b)^{*} \cup(a a a a)^{*} \uplus b^{*}$. Then $\left(i_{1}, i_{2}\right)=(0,0),\left(p_{1}, p_{2}\right)=$ $(4,2), \pi_{1}(L)=(a a)^{*}$ and $\pi_{2}(L)=b^{*}$.

Let $u, v \in \Sigma^{*}$. Then, $u$ is a subsequence 1 of $v$, denoted by $u \leqslant v$, if and only if $v \in u \amalg \Sigma^{*}$. The thereby given order is called the subsequence order. Let $L \subseteq \Sigma^{*}$. Then, we define (1) the upward closure $\uparrow L=L \amalg \Sigma^{*}=\left\{u \in \Sigma^{*}: \exists v \in L: v \leqslant u\right\}$; (2) the downward closure $\downarrow L=\left\{u \in \Sigma^{*}: u \amalg \Sigma^{*} \cap L \neq \varnothing\right\}=\left\{u \in \Sigma^{*}: \exists v \in\right.$ $L: u \leqslant v\}$; (3) the upward interior, denoted by $\circlearrowleft L$, as the largest upwardclosed set in $L$, i.e. the largest subset $U \subseteq L$ such that $\uparrow U=U$ and (4) the downward interior, denoted by $\Omega L$, as the largest downward-closed set in $L$, i.e., the largest subset $U \subseteq L$ such that $\downarrow U=U$. We have $\Omega L=\Sigma^{*} \backslash \uparrow\left(\Sigma^{*} \backslash L\right)$ and $\circlearrowleft L=\Sigma^{*} \backslash \downarrow\left(\Sigma^{*} \backslash L\right)$.

The following two results, which will be needed later, are from [1415.

Theorem 4. Let $U, V \subseteq \Sigma^{*}$ be commutative regular languages with index and period vectors $\left(i_{1}, \ldots, i_{k}\right),\left(j_{1}, \ldots, j_{k}\right)$ and $\left(p_{1}, \ldots, p_{k}\right),\left(q_{1}, \ldots, q_{k}\right)$. Then, the index vector of $U \amalg V$ is at most

$$
\left(i_{1}+j_{1}+\operatorname{lcm}\left(p_{1}, q_{1}\right)-1, \ldots, i_{k}+j_{k}+\operatorname{lcm}\left(p_{k}, q_{k}\right)-1\right)
$$

and the period vector is at most $\left(\operatorname{lcm}\left(p_{1}, q_{1}\right), \ldots, \operatorname{lcm}\left(p_{k}, q_{k}\right)\right) . S o, \operatorname{sc}(U \amalg V) \leqslant$ $\prod_{l=1}^{k}\left(i_{l}+j_{l}+2 \cdot \operatorname{lcm}\left(p_{l}, q_{l}\right)-1\right)$.

Theorem 5. Let $\Sigma=\left\{a_{1}, \ldots, a_{k}\right\}$. Suppose $L \subseteq \Sigma^{*}$ is commutative and regular with index vector $\left(i_{1}, \ldots, i_{k}\right)$ and period vector $\left(p_{1}, \ldots, p_{k}\right)$. Then, $\max \{\operatorname{sc}(\uparrow$ $L), \operatorname{sc}(\downarrow L), \operatorname{sc}(\circlearrowleft L), \operatorname{sc}(\Omega L)\} \leqslant \prod_{j=1}^{k}\left(i_{j}+p_{j}\right)$.

\footnotetext{
${ }^{1}$ Also called a scattered subword in the literature [1119].
} 


\section{Product-Form Minimal Automata}

As shown in Example 1, the minimal automaton, in general, does not equal the minimal commutative automaton. Here, we introduce the class of commutative regular languages for which both are isomorphic. The corresponding commutative languages are called languages with a minimal automaton of product-form, as the minimal commutative automaton is built with the Cartesian product.

Definition 6 (languages with product-form minimal automaton). $A$ commutative and regular language $L \subseteq \Sigma^{*}$ is said to have a minimal automaton of product-form, if $\mathcal{C}_{L}$ is isomorphic to $\mathcal{A}_{L}$.

If $|\Sigma|=1$, we see easily that $\mathcal{C}_{L}$ is the minimal deterministic and complete automaton.

Proposition 7. If $|\Sigma|=1$, then each commutative and regular $L \subseteq \Sigma^{*}$ has a minimal automaton of product-form. More generally, if $L \subseteq\{a\}^{*}$, then $L \amalg$ $(\Sigma \backslash\{a\})^{*}$ has a minimal automaton of product-form.

Apart from the unary languages, we give another example of a language with minimal automaton of product-form next.

Example 3. Let $L=(a a)^{*} \amalg(b b)^{*} \cup(a a a)^{*} \amalg b(b b)^{*}$ over $\Sigma=\{a, b\}$. See Figure 2 for the minimal commutative automaton. Here, the minimal commutative automaton equals the minimal automaton.

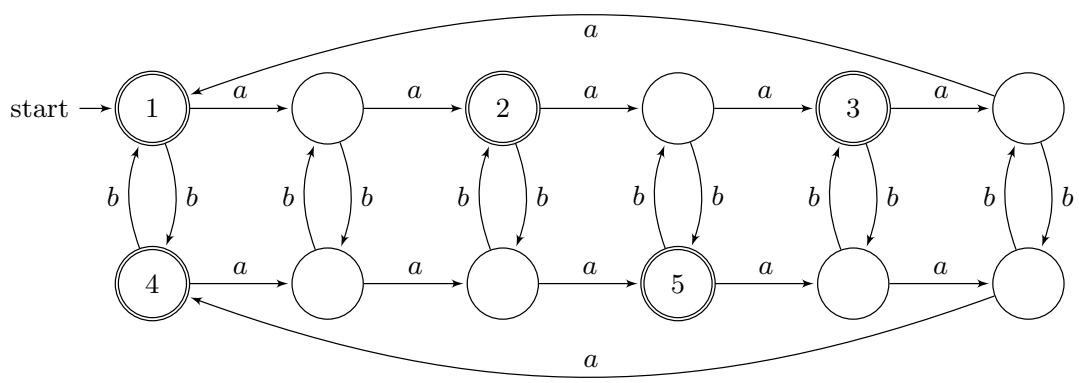

Fig. 2. $\mathcal{C}_{L}$ for $L=(a a)^{*} \uplus(b b)^{*} \cup(a a a)^{*} \uplus b(b b)^{*}$. Here $\mathcal{C}_{L}$ is isomorphic to $\mathcal{A}_{L}$.

However, the next proposition gives a strong necessary criterion for a commutative language to have a minimal automaton of product-form.

Proposition 8. If $L \subseteq \Sigma^{*}$ is commutative and regular with a minimal automaton of product-form, then $\mid\left\{x \in \Sigma \mid \pi_{\{x\}}(L)\right.$ is finite $\} \mid \leqslant 1$. So, $\pi_{\Gamma}(L)$ is infinite for $|\Gamma| \geqslant 2$, in particular no finite language over an at least binary alphabet is in this class. 
For example, $L=\{\varepsilon\}$ over $\Sigma$ does not have a minimal automaton of productform if $|\Sigma|>1$. Recall that the minimal automaton, as defined here, is always complete. Note that the converse of Proposition 8 is not true, as shown by $a a^{*}$ over $\Sigma=\{a, b\}$.

In the following statement, we give alternative characterizations for commutative languages with minimal automata of product-form.

Theorem 9, Let $L \subseteq \Sigma^{*}$ be a commutative regular language with index vector $\left(i_{1}, \ldots, i_{k}\right)$ and period vector $\left(p_{1}, \ldots, p_{k}\right)$. The following are equivalent:

1. the minimal automaton has product-form;

2. $\operatorname{sc}(L)=\prod_{j=1}^{k}\left(i_{j}+p_{j}\right)$;

3. $u \equiv_{L} v$ implies $\forall a \in \Sigma: a^{|u|_{a}} \equiv_{L} a^{|v|_{a}}$;

4. $u \equiv_{L} v$ if and only if $\forall a \in \Sigma: a^{|u|_{a}} \equiv_{L} a^{|v|_{a}}$.

Next, we give a way to construct commutative regular languages with minimal automata of product-form.

Lemma 10. Let $\Sigma=\left\{a_{1}, \ldots, a_{k}\right\}$ and, for $j \in\{1, \ldots, k\}, L_{j} \subseteq\left\{a_{j}\right\}^{*}$ be regular and infinite with index $i_{j}$ and period $p_{j}$. Then, $\operatorname{sc}\left(\bigsqcup_{j=1}^{k} L_{j}\right)=\prod_{j=1}^{k} \operatorname{sc}\left(L_{j}\right)=$ $\prod_{j=1}^{k}\left(i_{j}+p_{j}\right)$ and $\bigsqcup_{j=1}^{k} L_{j}$ has index vector $\left(i_{1}, \ldots, i_{k}\right)$ and period vector $\left(p_{1}, \ldots, p_{k}\right)$. With Thm. 9, $\bigsqcup_{j=1}^{k} L_{j}$ has a product-form minimal automaton.

In the next theorem and the following remark, we investigate closure properties of the class in question.

Theorem 11. The class of commutative regular languages with minimal automata of product-form is closed under left and right quotients and complementation. It is not closed under union, intersection and projection.

Remark 1. We have $a ш b^{*} \cap a^{*} ш b=a ш b$, showing, using Proposition 7 and 8 , that this class is not closed under intersection and by DeMorgan's laws, as we have closure under complementation, we also cannot have closure under union. Also, $L=a a^{*} \amalg b b^{*} \amalg c c^{*} \cup b b^{*} \amalg a^{*} \cup b^{*}$ has a minimal automaton of productform, but $\pi_{\{a, b\}}(L)=b b^{*} \amalg a^{*} \cup b^{*}$ is the language from Example 1. So, this class is also not closed under projection.

Theorem 12, Let $U, V \subseteq \Sigma^{*}$ be commutative regular languages with productform minimal automata with $\operatorname{sc}(U)=n$ and $\operatorname{sc}(V)=m$.

1. We have $\operatorname{sc}(U \amalg V) \leqslant 2 n m$ if $|\Sigma|>1$ and $\operatorname{sc}(U ш V) \leqslant n m$ if $|\Sigma|=1$. Furthermore, for any $\Sigma$, there exist $U, V$ as above such that $n m \leqslant \operatorname{sc}(U \sqcup V)$.

2. In the worst case, $n$ states are sufficient and necessary for a DFA to recognize $\uparrow U$. Similarly for the downward closure and interior operations.

3. In the worst case, $n$ states are sufficient and necessary for a DFA to recognize the projection of $U$.

4. In the worst case, $n m$ states are sufficient and necessary for a DFA to recognize $U \cap V$ or $U \cup V$. 
Remark 2. I do not know if the bound $2 \mathrm{~nm}$ stated in Theorem 1 for the shuffle operation is tight, but the next example shows that if we have a binary alphabet, we can find commutative languages with state complexities $n$ and $m$ and product-form minimal automata whose shuffle needs an automaton with strictly more than $n m$ states. A similar construction works for more than two letters. Let $p, q>11$ be two coprime numbers. Set $U=a \uplus b^{p-1}\left(b^{p}\right)^{*} \cup a^{p-1}\left(a^{p}\right)^{*} \amalg b b^{p-1}\left(b^{p}\right)^{*}$ and $V=b^{q-1}\left(b^{q}\right)^{*} \cup a^{q-1}\left(a^{q}\right)^{*} ш b b b^{q-1}\left(b^{q}\right)^{*}$. Then, using that shuffle distributes over union and a number-theoretical result from [27, Lemma 5.1], we find

$$
\begin{aligned}
& U ш V=a ш W \cup a^{p-1}\left(a^{p}\right)^{*} \amalg b W \cup \\
& \qquad a^{q}\left(a^{q}\right)^{*} \amalg b b W \cup a^{q-1+p-1}\left(a^{p}\right)^{*}\left(a^{q}\right)^{*} \amalg b b b W,
\end{aligned}
$$

where $a^{q-1+p-1}\left(a^{p}\right)^{*}\left(a^{q}\right)^{*}=F \cup a^{p q-1} a^{*}$ for some finite set $F \subseteq\left\{\varepsilon, a, \ldots, a^{p q-3}\right\}$ and $W=E \cup b^{p q-1} b^{*}$ for some $E \subseteq\left\{\varepsilon, b, \ldots, b^{p q-3}\right\}$. Note that by [27, Lemma 5.1] we have $a^{p q-2} ш b b b W \cap U ш V=\varnothing$. All languages involved have a productform minimal automaton. The minimal automaton for $U$ has $(2+p) \cdot(1+p)$ states, the minimal automaton for $V$ has $(1+q) \cdot(q+2)$ states and that for $U \amalg V$ has $2 p q \cdot(p q+3)$ states. As $(p-11)(q-11)>0$ we can deduce $(1+p)(2+p)(1+q)(2+q)<2(p q)^{2}<2 p q(p q+3)$.

\section{Partial Commutativity and Other Subclasses}

A partial commutation on $\Sigma$ is a symmetric and irreflexive relation $I \subseteq \Sigma \times \Sigma$, often called the independence relation. Of interest is the congruence $\sim_{I}$ generated on $\Sigma^{*}$ by the relation $\{(a b, b a) \mid(a, b) \in I\}$. A language $L \subseteq \Sigma^{*}$ is closed under $I$-commutation if $u \in L$ and $u \sim_{I} v$ implies $v \in L$. If $I=\{(a, b) \in \Sigma \times \Sigma \mid a \neq b\}$, then the languages closed under $I$-commutation are precisely the commutative languages.

Languages closed under some partial commutation relation have been extensively studied, see [10, also for further references, and in particular with relation to (Mazurkiewicz) trace theory [5]10]21], a formalism to describe the execution histories of concurrent programs.

Here, we will focus on the case that $(\Sigma \times \Sigma) \backslash I$ is transitive, i.e., if $u \ngtr_{I} v$ and $v \chi_{I} w$ implies $u \nsim_{I} w$. In this case, $(\Sigma \times \Sigma) \backslash I$ is an equivalence relation and we will write $\Sigma_{1}, \ldots, \Sigma_{k}$ for the different equivalence classes.

The reason to focus on this particular generalization is, as we will see later, that the definition of the minimal commutative automaton transfers to this more general setting without much difficulty.

To ease the notation, if we have a partial commutation relation as above with a corresponding partition $\Sigma=\Sigma_{1} \cup \ldots \Sigma_{k}$ of the alphabet, we also write $\mathcal{L}_{\Sigma_{1}, \ldots, \Sigma_{k}}$ for the class of languages closed under this partial commutation. Then, as is easily seen, we have $L \in \mathcal{L}_{\Sigma_{1}, \ldots, \Sigma_{k}}$ if and only if, for $x \in \Sigma_{i}, y \in \Sigma_{j}$ $(i \neq j)$ and each $u, v \in \Sigma^{*}$ we have uxyv $\in L \Leftrightarrow$ uyxv $\in L$. For example, $L$ is commutative if and only if $L \in \mathcal{L}_{\left\{a_{1}\right\}, \ldots,\left\{a_{k}\right\}}$ for $\Sigma=\left\{a_{1}, \ldots, a_{k}\right\}$. 


\subsection{The Canonical Automaton}

Here, we generalize our notion of commutative minimal automaton, Definition 1, to have uniform recognition devices for languages in $\mathcal{L}_{\Sigma_{1}, \ldots, \Sigma_{k}}$.

Definition 13. Let $\Sigma=\Sigma_{1} \cup \ldots \cup \Sigma_{k}$ be a partition and $L \subseteq \Sigma^{*}$. Set $\mathcal{C}_{L, \Sigma_{1}, \ldots, \Sigma_{k}}=$ $\left(\Sigma, S_{1} \times \ldots \times S_{k}, \delta, s_{0}, F\right)$ with, for $i \in\{1, \ldots, k\}, S_{i}=\left\{[u]_{\equiv_{L}} \mid u \in \Sigma_{i}^{*}\right\}$, $F=\left\{\left(\left[\pi_{\Sigma_{1}}(u)\right]_{\equiv_{L}}, \ldots,\left[\pi_{\Sigma_{k}}(u)\right]_{\equiv_{L}}\right) \mid u \in L\right\}, s_{0}=\left([\varepsilon]_{\equiv_{L}}, \ldots,[\varepsilon]_{\equiv_{L}}\right)$ and, for $x \in \Sigma_{i}$,

$$
\delta\left(\left(\left[u_{1}\right]_{\equiv_{L}}, \ldots,\left[u_{i}\right]_{\equiv_{L}}, \ldots,\left[u_{k}\right]_{\equiv_{L}}\right), x\right)=\left(\left[u_{1}\right]_{\equiv_{L}}, \ldots,\left[u_{i} x\right]_{\equiv_{L}}, \ldots,\left[u_{k}\right]_{\equiv_{L}}\right)
$$

with words $u_{j} \in \Sigma_{j}^{*}, j \in\{1, \ldots, k\}$. This is called the canonical automaton for the given $L$ with respect to $\Sigma=\Sigma_{1} \cup \ldots \cup \Sigma_{k}$.

Next, we show that the canonical automata recognize precisely the languages in $\mathcal{L}_{\Sigma_{1}, \ldots, \Sigma_{k}}$. Note that we have dropped the assumption of regularity of $L$.

Theorem 14. Let $L \subseteq \Sigma^{*}$ and $\Sigma=\Sigma_{1} \cup \ldots \cup \Sigma_{k}$ be a partition. Then,

1. $L \subseteq L\left(\mathcal{C}_{L, \Sigma_{1}, \ldots, \Sigma_{k}}\right)$ and $L\left(\mathcal{C}_{L, \Sigma_{1}, \ldots, \Sigma_{k}}\right) \in \mathcal{L}_{\Sigma_{1}, \ldots, \Sigma_{k}}$.

2. $L=L\left(\mathcal{C}_{L, \Sigma_{1}, \ldots, \Sigma_{k}}\right) \Leftrightarrow L \in \mathcal{L}_{\Sigma_{1}, \ldots, \Sigma_{k}}$.

3. Let $L \in \mathcal{L}_{\Sigma_{1}, \ldots, \Sigma_{k}}$. Then $L$ is regular if and only if $\mathcal{C}_{L, \Sigma_{1}, \ldots, \Sigma_{k}}$ is finite.

Also, used in defining a subclass in the next subsection, we will derive a canonical automaton for certain projected languages from $\mathcal{C}_{L, \Sigma_{1}, \ldots, \Sigma_{k}}$. Essentially, the next definition and proposition mean that if we only use one "coordinate" of $\mathcal{C}_{L, \Sigma_{1}, \ldots, \Sigma_{k}}$, then this recognizes a projection of $L$.

Definition 15. Let $i \in\{1, \ldots, k\}$ and $L \in \mathcal{L}_{\Sigma_{1}, \ldots, \Sigma_{k}}$. The canonical projection automaton (for $\Sigma_{i}$ ) is $\mathcal{C}_{L, \Sigma_{i}}=\left(\Sigma_{i}, S_{i}, \delta_{i},[\varepsilon]_{\equiv_{L}}, F_{i}\right)$ with $S_{i}=\left\{[u]_{\equiv_{L}} \mid u \in \Sigma_{i}^{*}\right\}$, $\delta_{i}\left([u]_{\equiv_{L}}, x\right)=[u x]_{\equiv_{L}}$ for $x \in \Sigma_{i}$ and $F_{i}=\left\{\left[\pi_{\Sigma_{i}}(u)\right]_{\equiv_{L}} \mid u \in L\right\}$.

Proposition 16. Let $L \in \mathcal{L}_{\Sigma_{1}, \ldots, \Sigma_{k}}$. Then, for $i \in\{1, \ldots, k\}, \pi_{\Sigma_{i}}(L)=L\left(\mathcal{C}_{L, \Sigma_{i}}\right)$.

\subsection{Subclasses in $\mathcal{L}_{\Sigma_{1}, \ldots, \Sigma_{k}}$}

Here, we investigate several subclasses of $\mathcal{L}_{\Sigma_{1}, \ldots, \Sigma_{k}}$. Recall that, for $L \subseteq \Sigma^{*}$, the minimal automaton of $L$ is denoted by $\mathcal{A}_{L}$.

Definition 17. Let $\Sigma=\Sigma_{1} \cup \ldots \cup \Sigma_{k}$ be a partition. Then, define the following classes of languages.

$\mathcal{L}_{1}=\left\{L \mid \mathcal{C}_{L, \Sigma_{1}, \ldots, \Sigma_{k}}\right.$ has a single final state and $\left.L=L\left(\mathcal{C}_{L, \Sigma_{1}, \ldots, \Sigma_{k}}\right).\right\}$,

$\mathcal{L}_{2}=\left\{L \mid L=\bigsqcup_{i=1}^{k} \pi_{\Sigma_{i}}(L)\right\}$,

$\mathcal{L}_{3}=\left\{L \mid L=L\left(\mathcal{C}_{L, \Sigma_{1}, \ldots, \Sigma_{k}}\right), \forall i \in\{1, \ldots, k\}: \mathcal{A}_{\pi_{\Sigma_{i}}(L)}\right.$ is isomorphic to $\left.\mathcal{C}_{L, \Sigma_{i}}\right\}$,

$\mathcal{L}_{4}=\left\{L \mid \mathcal{A}_{L}\right.$ is isomorphic to $\left.\mathcal{C}_{L, \Sigma_{1}, \ldots, \Sigma_{k}}\right\}$. 
First, we show that these are in fact subclasses of $\mathcal{L}_{\Sigma_{1}, \ldots, \Sigma_{k}}$.

Proposition 18. Let $\Sigma=\Sigma_{1} \cup \ldots \cup \Sigma_{k}$ be a partition. For each $i \in\{1,2,3,4\}$ we have $\mathcal{L}_{i} \subseteq \mathcal{L}_{\Sigma_{1}, \ldots, \Sigma_{k}}$.

Remark 3. Regarding $\mathcal{L}_{1}$, note that there exist languages $L=L\left(\mathcal{C}_{L, \Sigma_{1}, \ldots, \Sigma_{k}}\right)$ such that the minimal automaton has a single final state, but $\mathcal{C}_{L, \Sigma_{1}, \ldots, \Sigma_{k}}$ has more than one final state. For example, $L=\left\{\left.w \in\{a, b\}^{*}|| w\right|_{a}>0\right.$ or $\left.|w|_{b}>0\right\}$. However, if $\mathcal{C}_{L, \Sigma_{1}, \ldots, \Sigma_{k}}$ has a single final state, then the minimal automata also has only a single final state.

Example 4. Let $\Sigma=\Sigma_{1} \cup \Sigma_{2}$ with $\Sigma_{1}=\{a\}$ and $\Sigma_{2}=\{b\}$. Set $L=\left(a a(a a a)^{*} \amalg\right.$ $\left.b b(b b b)^{*}\right) \cup\left(a(a a a)^{*} \amalg b(b b b)^{*}\right)$. Then $L \in\left(\mathcal{L}_{3} \cap \mathcal{L}_{4}\right) \backslash \mathcal{L}_{2}$.

Example 5. Set $L=\left(a(a a a)^{*} ш b\right) \cup a a(a a a)^{*}$. Then $L \in \mathcal{L}_{3} \backslash \mathcal{L}_{4}$.

The languages in $\mathcal{L}_{1}$ arise in connection with the canonical automaton.

Proposition 19. Let $L \in \mathcal{L}_{\Sigma_{1}, \ldots, \Sigma_{k}}$ and $\mathcal{C}_{L, \Sigma_{1}, \ldots, \Sigma_{k}}=\left(\Sigma, S_{1} \times \ldots \times S_{k}, \delta, s_{0}, F\right)$. Then, for all $s \in S_{1} \times \ldots \times S_{k},\left\{w \in \Sigma^{*} \mid \delta\left(s_{0}, w\right)=s\right\} \in \mathcal{L}_{1}$.

Next, we give alternative characterization for $\mathcal{L}_{2}, \mathcal{L}_{3}$ and $\mathcal{L}_{4}$.

Theorem [20, Let $L \in \mathcal{L}_{\Sigma_{1}, \ldots, \Sigma_{k}}$. Then,

1. $L \in \mathcal{L}_{2}$ if and only if, for each $w \in \Sigma^{*}$, the following is true:

$$
w \in L \Leftrightarrow \forall i \in\{1, \ldots, k\}: \pi_{\Sigma_{i}}(w) \in \pi_{\Sigma_{i}}(L)
$$

2. $L \in \mathcal{L}_{3}$ if and only if, for all $i \in\{1, \ldots, k\}$ and $u \in \Sigma_{i}^{*}$, we have

$$
[u]_{\equiv_{L}} \cap \Sigma_{i}^{*}=[u]_{\equiv_{\pi_{\Sigma_{i}}(L)}} \cap \Sigma_{i}^{*}
$$

3. $L \in \mathcal{L}_{4}$ if and only if, for each $u, v \in \Sigma^{*}$,

$$
u \equiv_{L} v \Leftrightarrow \forall i \in\{1, \ldots, k\}: \pi_{\Sigma_{i}}(u) \equiv_{L} \pi_{\Sigma_{i}}(v) .
$$

Example 6. Let $L_{1}$ be the language from Example 3. Set $L_{2}=a_{1} ш a_{2}=$ $\left\{a_{1} a_{2}, a_{2} a_{1}\right\}$. Both of their letters commute for the partition $\left\{a_{1}, a_{2}\right\}=\left\{a_{1}\right\} \cup$ $\left\{a_{2}\right\}$. Then, $L_{1} \in \mathcal{L}_{4} \backslash \mathcal{L}_{3}$ and $L_{2} \in \mathcal{L}_{1} \backslash \mathcal{L}_{4}$.

Finally, in Theorem 21, we establish inclusion relations, which are all proper, between $\mathcal{L}_{1}, \mathcal{L}_{2}$ and $\mathcal{L}_{3}$, also see Figure 3 .

Theorem [21. We have $\mathcal{L}_{1} \subsetneq \mathcal{L}_{2} \subsetneq \mathcal{L}_{3}$.

Remark 4. Theorem 21 and Example 6 show that $\mathcal{L}_{4}$ is incomparable to each of the other language classes with respect to inclusion. 


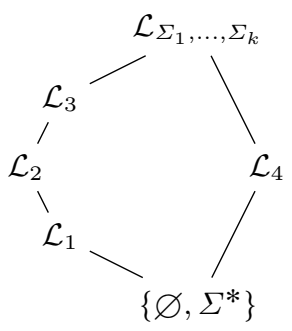

Fig. 3. Inclusion relations between the language classes.

\section{Conclusion}

The language class of commutative regular languages with minimal automata of product-form behaves well with respect to the descriptional complexity measure of state complexity for certain operations, see Table 2, and Lemma 10 allows us to construct infinitely many commutative regular languages with product-form minimal automaton. The investigation started could be carried out for other operations and measures of descriptional complexity as well. Likewise, as done in [89] for commutative and more general partial commutativity conditions, it might be interesting if the learning algorithms given there could be improved for the language class introduced.

Lastly, if the bound $2 \mathrm{~nm}$ for shuffle is tight is an open problem. Remark 2 shows that the bound $\mathrm{nm}$ is not sufficient, however, giving an infinite family of commutative regular languages with minimal automata of product-form attaining the bound $2 \mathrm{~nm}$ for shuffle is an open problem.

Acknowledgement. I thank the anonymous referees of [14] (the extended version of [15]), whose feedback also helped in the present work. I also sincerely thank the referees of the present submission, which helped me alot in identifying unclear or ungrammatical formulations and a missing definition.

\section{References}

1. Brzozowski, J.A., Jirásková, G., Liu, B., Rajasekaran, A., Szykuła, M.: On the state complexity of the shuffle of regular languages. In: Câmpeanu, C., Manea, F., Shallit, J.O. (eds.) DCFS 2016. LNCS, vol. 9777, pp. 73-86. Springer (2016)

2. Brzozowski, J.A., Simon, I.: Characterizations of locally testable events. Discrete Mathematics 4(3), 243-271 (1973)

3. Campbell, R.H., Habermann, A.N.: The specification of process synchronization by path expressions. In: Gelenbe, E., Kaiser, C. (eds.) Operating Systems OS. LNCS, vol. 16, pp. 89-102. Springer (1974)

4. Câmpeanu, C., Salomaa, K., Yu, S.: Tight lower bound for the state complexity of shuffle of regular languages. J. Autom. Lang. Comb. 7(3), 303-310 (2002)

5. Diekert, V., Rozenberg, G. (eds.): The Book of Traces. World Scientific (1995)

6. Ehrenfeucht, A., Haussler, D., Rozenberg, G.: On regularity of context-free languages. Theor. Comput. Sci. 27, 311-332 (1983) 
7. Gao, Y., Moreira, N., Reis, R., Yu, S.: A survey on operational state complexity. Journal of Automata, Languages and Combinatorics 21(4), 251-310 (2017)

8. Gómez, A.C., Alvarez, G.I.: Learning commutative regular languages. In: Clark, A., Coste, F., Miclet, L. (eds.) ICGI. LNCS, vol. 5278, pp. 71-83. Springer (2008)

9. Gómez, A.C.: Inferring regular trace languages from positive and negative samples. In: Sempere, J.M., García, P. (eds.) ICGI. LNCS, vol. 6339, pp. 11-23. Springer (2010)

10. Gómez, A.C., Guaiana, G., Pin, J.: Regular languages and partial commutations. Inf. Comput. 230, 76-96 (2013)

11. Gruber, H., Holzer, M., Kutrib, M.: The size of Higman-Haines sets. Theor. Comput. Sci. 387(2), 167-176 (2007)

12. Gruber, H., Holzer, M., Kutrib, M.: More on the size of Higman-Haines sets: Effective constructions. Fundam. Informaticae 91(1), 105-121 (2009)

13. Héam, P.: On shuffle ideals. RAIRO Theor. Inform. Appl. 36(4), 359-384 (2002)

14. Hoffmann, S.: Commutative regular languages - properties and state complexity. Information and Computation (to appear)

15. Hoffmann, S.: Commutative regular languages - properties and state complexity. In: Ciric, M., Droste, M., Pin, J. (eds.) CAI 2019. LNCS, vol. 11545, pp. 151-163. Springer (2019)

16. Hoffmann, S.: State complexity investigations on commutative languages - the upward and downward closure, commutative aperiodic and commutative group languages. In: Han, Y.S., Ko, S.K. (eds.) DCFS 2021, Proceedings. LNCS, Springer (2021)

17. Hoffmann, S.: State complexity of projection on languages recognized by permutation automata and commuting letters. In: Moreira, N., Reis, R. (eds.) DLT 2021, Porto, Portugal, August 16-20, 2021, Proceedings. LNCS, vol. 12811, pp. 192-203. Springer (2021)

18. Jirásková, G., Masopust, T.: On a structural property in the state complexity of projected regular languages. Theor. Comput. Sci. 449, 93-105 (2012)

19. Karandikar, P., Niewerth, M., Schnoebelen, P.: On the state complexity of closures and interiors of regular languages with subwords and superwords. Theor. Comput. Sci. 610, 91-107 (2016)

20. Maslov, A.N.: Estimates of the number of states of finite automata. Dokl. Akad. Nauk SSSR 194(6), 1266-1268 (1970)

21. Mazurkiewicz, A.: Concurrent program schemes and their interpretations. Tech. rep., DAIMI Report Series, 6(78) (1977)

22. Mazurkiewicz, A.W.: Parallel recursive program schemes. In: Becvár, J. (ed.) MFCS 1975. LNCS, vol. 32, pp. 75-87. Springer (1975)

23. Okhotin, A.: On the state complexity of scattered substrings and superstrings. Fundam. Informaticae 99(3), 325-338 (2010)

24. Pighizzini, G., Shallit, J.: Unary language operations, state complexity and jacobsthal's function. Int. J. Found. Comput. Sci. 13(1), 145-159 (2002)

25. Shaw, A.C.: Software descriptions with flow expressions. IEEE Trans. Softw. Eng. 4, 242-254 (1978)

26. Wong, K.: On the complexity of projections of discrete-event systems. In: Proc. of WODES 1998, Cagliari, Italy. pp. 201—206 (1998)

27. Yu, S., Zhuang, Q., Salomaa, K.: The state complexities of some basic operations on regular languages. Theor. Comput. Sci. 125(2), 315-328 (Mar 1994) 


\section{A Proofs for Section 2 (Preliminaries)}

Remark 5. Let $L \subseteq \Sigma^{*}$ be commutative and $\mathcal{C}_{L}=\left(\Sigma, S_{1} \times \ldots \times S_{k}, \delta, s_{0}, F\right)$ be the minimal commutative automaton. Note that, by the definition of the transition function in Definition 1, we have, for all $u, v \in \Sigma^{*}$,

$$
\delta\left(s_{0}, u\right)=\delta\left(s_{0}, v\right) \Leftrightarrow \forall j \in\{1, \ldots, k\}: \pi_{j}(u) \equiv_{L} \pi_{j}(v) .
$$

For a commutative language $L \subseteq \Sigma^{*}$, the Nerode right- and left-congruence and the syntactic congruence coincide. So, as $[u]_{\equiv_{L}}=\left[\pi_{1}(u) \cdots \pi_{k}(u)\right]_{\equiv_{L}}=$ $\left[\pi_{1}(u)\right]_{\equiv_{L}} \cdots\left[\pi_{k}(u)\right]_{\equiv_{L}}$ we have:

$$
\forall j \in\{1, \ldots, k\}: \pi_{j}(u) \equiv_{L} \pi_{j}(v) \Rightarrow u \equiv_{L} v .
$$

\section{B Proofs for Section 3 (Product-Form Minimal Automata)}

Proposition 8, If $L \subseteq \Sigma^{*}$ is commutative and regular with a minimal automaton of product-form, then $\mid\left\{x \in \Sigma \mid \pi_{\{x\}}(L)\right.$ is finite $\} \mid \leqslant 1$. So, $\pi_{\Gamma}(L)$ is infinite for $|\Gamma| \geqslant 2$, in particular no finite language over an at least binary alphabet is in this class.

Proof. Suppose we have two distinct $j, j^{\prime} \in\{1, \ldots, k\}$ such that $\pi_{j}(L)$ and $\pi_{j^{\prime}}(L)$ are finite. Set $N=\max +1$. Then $a_{j}^{N} \equiv_{L} a_{j^{\prime}}^{N}$ and, as $N>0$, this implies that the minimal commutative automaton has strictly more states than the minimal deterministic automaton.

For the last sentence, note that if $a \in \Gamma$, then $\pi_{\{a\}}(L)=\pi_{\{a\}}\left(\pi_{\Gamma}(L)\right)$. Hence, if $\pi_{\Gamma}(L)$ is finite with $|\Gamma| \geqslant 2$, then at least two one-letter projection languages would be finite too. Hence, with the previous claim, if $L$ is commutative and regular, it does not have a minimal automaton of product-form in this case.

Theorem 9. Let $L \subseteq \Sigma^{*}$ be a commutative regular language with index vector $\left(i_{1}, \ldots, i_{k}\right)$ and period vector $\left(p_{1}, \ldots, p_{k}\right)$. The following are equivalent:

1. the minimal automaton has product-form;

2. $\operatorname{sc}(L)=\prod_{j=1}^{k}\left(i_{j}+p_{j}\right)$;

3. $u \equiv_{L} v$ implies $\forall a \in \Sigma: a^{|u|_{a}} \equiv_{L} a^{|v|_{a}}$;

4. $u \equiv_{L} v$ if and only if $\forall a \in \Sigma: a^{|u|_{a}} \equiv_{L} a^{|v|_{a}}$.

Proof. First, suppose $\mathcal{C}_{L}$ is isomorphic to $\mathcal{A}_{L}$. Then, by the definition of the state complexity, we find $\operatorname{sc}(L)=\prod_{j=1}^{k}\left(i_{j}+p_{j}\right)$. Conversely, suppose $\operatorname{sc}(L)=$ $\prod_{j=1}^{k}\left(i_{j}+p_{j}\right)$. As $L$ is commutative, we have $L=L\left(\mathcal{C}_{L}\right)$. Every automaton recognizing $L$ in which all states are reachable from the start state can be mapped surjectively onto $\mathcal{A}_{L}$, see [Hop71,Koz97]. In particular, this holds true for $\mathcal{C}_{L}$. By 
finiteness, as both have the same number of states, this must be an isomorphism. Hence, the first two conditions are equivalent

That the last condition is equivalent to the first is shown, in a more general context without referring to any previous result, in Theorem 20, as the case of commutative languages corresponds to the case $\Sigma_{1}=\left\{a_{1}\right\}, \ldots, \Sigma_{k}=\left\{a_{k}\right\}$ with the notation from Section 4 .

That the third condition is equivalent to the last condition follows as for a commutative language we have $[u]_{\equiv_{L}}=\left[a_{1}^{|u|_{a_{1}}} \cdots a_{k}^{|u|_{a_{k}}}\right]_{\equiv_{L}}$, so if $\forall a \in \Sigma$ : $a^{|u|_{a}} \equiv_{L} a^{|v|_{a}}$, then $u \equiv_{L} v$ is always valid for commutative languages.

Let $L \subseteq \Sigma^{*}$. Next, we will need the following equations:

$$
u \in \bigsqcup_{i=1}^{k} \pi_{i}(L) \Leftrightarrow \forall i \in\{1, \ldots, k\}: \pi_{i}(u) \in \pi_{i}(L),
$$

and

$$
L \subseteq \bigsqcup_{i=1}^{k} \pi_{i}(L)
$$

Lemma 22. Let $L \subseteq \Sigma^{*}$ be a commutative language with minimal commutative automaton $\mathcal{C}_{L}=\left(\Sigma, S_{1} \times \ldots \times S_{k}, \delta, s_{0}, F\right)$, index vector $\left(i_{1}, \ldots, i_{k}\right)$ and period vector $\left(p_{1}, \ldots, p_{k}\right)$. Set $n=|F|$. Suppose $F=\left\{\left(s_{1}^{(l)}, \ldots, s_{k}^{(l)}\right) \mid l \in\{1, \ldots, n\}\right\}$ and set, for $l \in\{1, \ldots, n\}, L^{(l)}=\left\{w \in \Sigma^{*} \mid \delta\left(s_{0}, w\right)=\left(s_{1}^{(l)}, \ldots, s_{k}^{(l)}\right)\right\}$, and $L_{j}^{(l)}=\pi_{j}\left(L^{(l)}\right), j \in\{1, \ldots, k\}$. Then,

$$
L=\bigcup_{l=1}^{n} L^{(l)} \text { and } L^{(l)}=\bigsqcup_{j=1}^{k} L_{j}^{(l)} .
$$

For $j \in\{1, \ldots, k\}$, set $\mathcal{C}_{L,\left\{a_{j}\right\}}=\left(\left\{a_{j}\right\}, S_{j}, \delta_{j},[\varepsilon]_{\equiv_{L}}, F_{j}\right)$ with

$$
\delta_{j}\left(\left[a_{j}^{m}\right]_{\equiv_{L}}, a_{j}\right)=\left[a_{j}^{m+1}\right]_{\equiv_{L}} \text { and } F_{j}=\left\{s_{j}^{(1)}, \ldots, s_{j}^{(n)}\right\}
$$

for $\left[a_{j}^{m}\right]_{\equiv_{L}} \in S_{j}, m \geqslant 0$. Then,

$$
L_{j}^{(l)}=L\left(\left(\left\{a_{j}\right\}, S_{j}, \delta_{j},[\varepsilon]_{\equiv_{L}},\left\{s_{j}^{(l)}\right\}\right) \text { and } L\left(\mathcal{C}_{L,\left\{a_{j}\right\}}\right)=\pi_{j}(L)=\bigcup_{l=1}^{n} L_{j}^{(l)} .\right.
$$

for $l \in\{1, \ldots, n\}$ and the automata $\mathcal{C}_{L,\left\{a_{j}\right\}}$ have index $i_{j}$ and period $p_{j}$.

Proof. As every accepted word drives $\mathcal{C}_{L}$ into some final state, we have $L=$ $\bigcup_{l=1}^{n} L^{(l)}$. Next, we show the other claims.

Claim: Let $l \in\{1, \ldots, n\}$. Then $L^{(l)}=\bigsqcup_{j=1}^{k} L_{j}^{(l)}$.

Proof of the Claim: By Equation (4), we have $L^{(l)} \subseteq \bigsqcup_{j=1}^{k} L_{j}^{(l)}$. If $w \in \bigsqcup_{j=1}^{k} L_{j}^{(l)}$, then, for $j \in\{1, \ldots, k\}$, there exists $u_{j} \in L_{j}^{(l)}$ such that $\pi_{j}(w)=\pi_{j}\left(u_{j}\right)$. Hence, by Remark 5, $\delta\left(s_{0}, w\right)=\delta\left(s_{0}, u_{1} \cdots u_{j}\right)=$ $\left(s_{1}^{(l)}, \ldots, s_{k}^{(l)}\right)$, and so $w \in L^{(l)}$. [End, Proof of the Claim] 
Claim: Let $l \in\{1, \ldots, n\}$ and $j \in\{1, \ldots, k\}$. Then

$$
L_{j}^{(l)}=L\left(\left(\left\{a_{j}\right\}, S_{j}, \delta_{j},[\varepsilon]_{\equiv_{L}},\left\{s_{j}^{(l)}\right\}\right) .\right.
$$

Proof of the Claim: By Remark 5 , for $u \in a_{j}^{*}$,

$$
\begin{aligned}
& \delta_{j}\left([\varepsilon]_{\equiv_{L}}, u\right)=s_{j}^{(l)} \\
& \Leftrightarrow[u]_{\equiv_{L}}=s_{j}^{(l)} \\
& \Leftrightarrow \exists w \in L^{(l)}: u \equiv_{L} \pi_{j}(w) \\
& \Leftrightarrow \exists w \in L^{(l)}: \delta\left(s_{0}, \pi_{1}(w) \cdots \pi_{j-1}(w) u \pi_{j+1}(w) \cdots \pi_{k}(w)\right)=\delta\left(s_{0}, w\right) \\
& \Leftrightarrow \exists w \in \Sigma^{*}: \delta\left(s_{0}, \pi_{1}(w) \cdots \pi_{j-1}(w) u \pi_{j+1}(w) \cdots \pi_{k}(w)\right)=\left(s_{1}^{(l)}, \ldots, s_{k}^{(l)}\right) \\
& \Leftrightarrow u \in L_{j}^{(l)} .
\end{aligned}
$$

And this shows the claim. [End, Proof of the Claim]

As $F_{j}=\left\{s_{j}^{(1)}, \ldots, s_{j}^{(n)}\right\}$, we find $L\left(\mathcal{C}_{L,\left\{a_{j}\right\}}\right)=\bigcup_{l=1}^{n} L_{j}^{(l)}$ and, with the previous equations and $L_{j}^{(l)} \subseteq a_{j}^{*}$,

$$
\pi_{j}(L)=\pi_{j}\left(\bigcup_{l=1}^{n} \bigsqcup_{i=1}^{k} L_{i}^{(l)}\right)=\bigcup_{l=1}^{n} \pi_{j}\left(\bigsqcup_{i=1}^{k} L_{i}^{(l)}\right)=\bigcup_{l=1}^{n} L_{j}^{(l)} .
$$

Lastly, that the automata $\mathcal{C}_{L,\left\{a_{j}\right\}}$ have index $i_{j}$ and period $p_{j}$ is implied as the Nerode right-congruence classes $\left[a_{j}^{m}\right]_{\equiv_{L}}, m \geqslant 0$ are the states of these automata. So, we have shown all equations in the statement.

Lemma 23. Let $L \subseteq \Sigma^{*}$ be a language with $L=\bigsqcup_{i=1}^{k} \pi_{i}(L)$. Then, for each $j \in\{1, \ldots k\}$ and $n, m \geqslant 0$, we have $a_{j}^{m} \equiv_{L} a_{j}^{n} \Leftrightarrow a_{j}^{m} \equiv_{\pi_{j}(L)} a_{j}^{n}$, where on the right side the equivalence is considered with respect to the unary alphabet $\left\{a_{j}\right\}$.

Proof. In the next equations, we will write " $\Leftrightarrow$ " to mean two formulas are semantically equivalent, and " $\leftrightarrow$ " as an equivalence in the formula itself.

By assumption and Equation (3), we have

$$
w \in L \Leftrightarrow \forall r \in\{1, \ldots k\}: \pi_{r}(w) \in \pi_{r}(L) .
$$

Now, let $j \in\{1, \ldots k\}$ be fixed and $n, m \geqslant 0$. We have

$$
a_{j}^{m} \equiv_{L} a_{j}^{n} \Leftrightarrow \forall x \in \Sigma^{*}: a_{j}^{m} x \in L \leftrightarrow a_{j}^{n} x \in L .
$$

By Equation (5), this is equivalent to

$$
\begin{aligned}
\forall x \in \Sigma^{*}:\left(\forall r \in\{1, \ldots, k\}: \pi_{r}\left(a_{j}^{m} x\right)\right. & \left.\in \pi_{r}(L)\right) \\
& \leftrightarrow\left(\forall r \in\{1, \ldots, k\}: \pi_{r}\left(a_{j}^{n} x\right) \in \pi_{r}(L)\right) .
\end{aligned}
$$

Claim: Equation (6) is equivalent to

$$
\forall x \in \Sigma^{*}: \pi_{j}\left(a_{j}^{m} x\right) \in \pi_{j}(L) \leftrightarrow \pi_{j}\left(a_{j}^{n} x\right) \in \pi_{j}(L) .
$$


Proof of the Claim: First, suppose Equation (6) holds true and suppose, for $x \in \Sigma^{*}$, we have $\pi_{j}\left(a_{j}^{m} x\right) \in \pi_{j}(L)$. Choose, for $r \neq j$, words $u_{r} \in$ $\pi_{r}(L)$, which is possible as $L=\bigsqcup_{r=1}^{k} \pi_{r}(L)$. Set $u_{j}=\pi_{j}(x)$ and $y=$ $u_{1} \cdots u_{k}$. Then, for all $r \in\{1, \ldots, k\}$, as $u_{r} \in a_{r}^{*}$, and hence

$$
\pi_{r}\left(a_{j}^{m} y\right)= \begin{cases}\pi_{r}\left(a_{j}^{m} x\right) & \text { if } r=j \\ \pi_{r}\left(u_{r}\right) & \text { if } r \neq j\end{cases}
$$

we have $\pi_{r}\left(a_{j}^{m} y\right) \in \pi_{r}(L)$. So, by Equation (6), we can deduce that, for all $r \in\{1, \ldots, k\}$, we have $\pi_{r}\left(a_{j}^{n} y\right) \in \pi_{r}(L)$. In particular, we find $\pi_{j}\left(a_{j}^{n} y\right) \in \pi_{j}(L)$. But $\pi_{j}\left(a_{j}^{n} y\right)=\pi_{j}\left(a_{j}^{n}\right) \pi_{j}(y)=\pi_{j}\left(a_{j}^{n}\right) \pi_{j}(x)=\pi_{j}\left(a_{j}^{n} x\right)$. So, $\pi_{j}\left(a_{j}^{n} x\right) \in \pi_{j}(L)$. Similarly, we can show that, for all $x \in \Sigma^{*}$, $\pi_{j}\left(a_{j}^{n} x\right) \in \pi_{j}(L)$ implies $\pi_{j}\left(a^{m} x\right) \in \pi_{j}(L)$.

Conversely, now suppose, for each $x \in \Sigma^{*}$, we have

$$
\pi_{j}\left(a_{j}^{m} x\right) \in \pi_{j}(L) \leftrightarrow \pi_{j}\left(a_{j}^{n} x\right) \in \pi_{j}(L) .
$$

Let $x \in \Sigma^{*}$ and suppose, for all $r \in\{1, \ldots, k\}$, we have $\pi_{r}\left(a_{j}^{m} x\right) \in$ $\pi_{r}(L)$. As, for $r \neq j$, we have $\pi_{r}\left(a_{j}^{m} x\right)=\pi_{r}(x)=\pi_{r}\left(a_{j}^{n} x\right)$ and, as, by assumption, the condition $\pi_{j}\left(a_{j}^{m} x\right) \in \pi_{j}(L)$ implies $\pi_{j}\left(a_{j}^{n} x\right) \in \pi_{j}(L)$, we find that, for all $r \in\{1, \ldots, k\}$, we have $\pi_{r}\left(a_{j}^{n} x\right) \in \pi_{r}(L)$. Similarly, we can show the other implication in Equation (6]). [End, Proof of the Claim]

So, by the previous claim, Equation (6) simplifies to

$$
\begin{aligned}
& \forall x \in \Sigma^{*}: \pi_{j}\left(a_{j}^{m} x\right) \in \pi_{j}(L) \leftrightarrow \pi_{j}\left(a_{j}^{n} x\right) \in \pi_{j}(L) \\
& \Leftrightarrow \forall x \in\left\{a_{j}\right\}^{*}: a_{j}^{m} x \in \pi_{j}(L) \leftrightarrow a_{j}^{n} x \in \pi_{j}(L) \\
& \Leftrightarrow a_{j}^{m} \equiv \pi_{j}(L) a_{j}^{n},
\end{aligned}
$$

and we have shown the statement.

Lemma 10, Let $\Sigma=\left\{a_{1}, \ldots, a_{k}\right\}$ and, for $j \in\{1, \ldots, k\}, L_{j} \subseteq\left\{a_{j}\right\}^{*}$ be regular and infinite with index $i_{j}$ and period $p_{j}$. Then, $\operatorname{sc}\left(\bigsqcup_{j=1}^{k} L_{j}\right)=\prod_{j=1}^{k} \operatorname{sc}\left(L_{j}\right)=$ $\prod_{j=1}^{k}\left(i_{j}+p_{j}\right)$ and $\bigsqcup_{j=1}^{k} L_{j}$ has index vector $\left(i_{1}, \ldots, i_{k}\right)$ and period vector $\left(p_{1}, \ldots, p_{k}\right)$. With Thm. [9, $\bigsqcup_{j=1}^{k} L_{j}$ has a product-form minimal automaton.

Proof. Set $L=\bigsqcup_{j=1}^{k} L_{j}$. Let $u, v \in \Sigma^{*}$ be two words such that

$$
u=a_{1}^{n_{1}} \cdot \ldots \cdot a_{k}^{n_{k}} \text { and } v=a_{1}^{m_{1}} \cdot \ldots \cdot a_{k}^{n_{k}} .
$$

with $0 \leqslant n_{j}, m_{j}<\operatorname{sc}\left(L_{j}\right), j \in\{1, \ldots, k\}$. Suppose there exists $r \in\{1, \ldots, k\}$ such that $n_{r} \neq m_{r}$. As $L_{r}$ is unary and $\max \left\{n_{r}, m_{r}\right\}<\operatorname{sc}\left(L_{r}\right)$,

$$
\varepsilon, a_{r}, a_{r}^{2}, \ldots, a_{r}^{\max \left\{n_{r}, m_{r}\right\}-1}
$$

are represenatives of distinct Nerode right-equivalence classes of $L_{r}$. Hence, there exists $l_{r}>0$ such that, without loss of generality,

$$
a_{r}^{n_{r}+l_{r}} \in L_{r} \text { and } a_{r}^{m_{r}+l_{r}} \notin L_{r} .
$$


As all the $L_{j}, j \in\{1, \ldots, k\}$, are infinite, there exists $l_{j} \geqslant 0$ for $j \in\{1, \ldots, k\} \backslash\{r\}$ such that $a_{j}^{n_{j}+l_{j}} \in L_{j}$. Then,

$$
a_{1}^{n_{1}+l_{1}} \cdot \ldots \cdot a_{k}^{n_{k}+l_{k}} \in L
$$

And, as $a_{r}^{m_{r}+l_{r}} \notin L_{r}$, we find

$$
a_{1}^{m_{1}+l_{1}} \cdot \ldots \cdot a_{k}^{m_{k}+l_{k}} \notin L
$$

Set $x=a_{1}^{l_{1}} \cdot \ldots \cdot a_{k}^{l_{k}}$. Then, as $L$ is a commutative language,

$$
u x \in L \text { and } v x \notin L,
$$

i.e., $u \not_{L} v$. Hence, all words of the form $a_{1}^{n_{1}} \cdot \ldots \cdot a_{k}^{n_{k}}$ with $0 \leqslant n_{j}<\operatorname{sc}\left(L_{j}\right)$ are pairwise non-equivalent. So, $\prod_{j=1}^{k} \operatorname{sc}\left(L_{j}\right) \leqslant \operatorname{sc}(L)$. Let $j \in\{1, \ldots, k\}$ and $\mathcal{A}_{L_{j}}=\left(\left\{a_{j}\right\}, Q_{L_{j}}, \delta_{L_{j}},[\varepsilon]_{\equiv_{L_{j}}}, F_{L_{j}}\right)$ be the minimal automaton for $L_{j}$. Also, let $\mathcal{C}_{L}=\left(\Sigma, S_{1} \times \ldots \times S_{k}, \delta, s_{0}, F\right)$ be the minimal commutative automaton from Definition 1 and consider the automaton $\mathcal{C}_{L,\left\{a_{j}\right\}}=\left(\left\{a_{j}\right\}, S_{j}, \delta_{j},[\varepsilon]_{\equiv_{L}}, F_{j}\right)$ from Lemma 22 By Theorem 2, we have $L=L\left(\mathcal{C}_{L}\right)$. Hence, $\operatorname{sc}(L) \leqslant \prod_{j=1}^{k}\left|S_{j}\right|$. By Lemma 23, as $L_{j}=\pi_{j}(L)$, the map

$$
\left[a_{j}^{n}\right]_{\equiv_{L}} \mapsto\left[a_{j}^{n}\right]_{\equiv_{L_{j}}}
$$

is a well-defined isomorphism between $\mathcal{A}_{L_{j}}$ and $\mathcal{C}_{L,\left\{a_{j}\right\}}$. So, $\left|S_{j}\right|=\operatorname{sc}\left(L_{j}\right)$. Hence,

$$
\operatorname{sc}(L) \leqslant \prod_{j=1}^{k} \operatorname{sc}\left(L_{j}\right)
$$

and, combining everything, $\operatorname{sc}(L)=\prod_{j=1}^{k} \operatorname{sc}\left(L_{j}\right)$. The entry of the index and period vector of $L$ for $j \in\{1, \ldots, k\}$ is the index and period of the unary automaton $\mathcal{C}_{L,\left\{a_{j}\right\}}$. As $\mathcal{C}_{L,\left\{a_{j}\right\}}$ and $\mathcal{A}_{L_{j}}$ are isomorphic, they have the same index and period. So, all claims of the statement are shown.

Theorem 11. The class of commutative regular languages with minimal automata of product-form is closed under left and right quotients and complementation. It is not closed under union, intersection and projection.

Proof. As for every language $L \subseteq \Sigma^{*}$, the state complexity of $L$ and the complement of $L$ are equal (recall we are only concerned with complete automata here) and $\mathcal{A}_{L}$ is minimal for both languages, closure of the class under complementation follows.

For commutative languages, left and right quotients give the same sets, i.e., $u^{-1} L=L u^{-1}$, hence it is sufficient to show closure under left quotients.

Now, if $\mathcal{A}_{L}=\left(\Sigma, Q_{L}, \delta_{L},[\varepsilon]_{\equiv_{L}}, F_{L}\right)$ is the minimal automaton, then, for $u \in \Sigma^{*}, \mathcal{B}=\left(\Sigma, Q_{L}, \delta_{L},[u]_{\equiv_{L}}, F_{L}\right)$ recognizes $u^{-1} L$. In fact, as for every $[u]_{\equiv_{L}} \neq$ $[v]_{\equiv_{L}}$, there exists $x \in \Sigma^{*}$ such that $u x \in L$ and $v x \notin L$, or vice versa. So, we 
have $[u x]_{\equiv_{L}} \in F_{L}$ and $[v x]_{\equiv_{L}} \notin F_{L}$, or vice versa. This implies, after discarding states not reachable from the start state, that $\mathcal{B}$ is isomorphic to the minimal automaton of $u^{-1} L$. Now, observe that $[w]_{\equiv_{L}}$ in $\mathcal{A}_{L}$ corresponds to the state $\left(\left[a_{1}^{|w|_{a_{1}}}\right]_{\equiv_{L}}, \ldots,\left[a_{k}^{|w|_{a_{k}}}\right]_{\equiv_{L}}\right)$ in $\mathcal{C}_{L}$. So, $\mathcal{B}$ is of product-form, which gives the claim. Note that, for every $j \in\{1, \ldots, k\}$, the index for the letter $a_{j}$ of $u^{-1} L$ is $\max \left\{i_{j}-\right.$ $\left.|u|_{a_{j}}, 0\right\}$ and the period for the letter $a_{j}$ is the same.

See Remark 1 for examples of languages which show that the class considered is not closed under union, intersection and projection.

Theorem 12, Let $U, V \subseteq \Sigma^{*}$ be commutative regular languages with productform minimal automata with $\operatorname{sc}(U)=n$ and $\operatorname{sc}(V)=m$.

1. We have $\operatorname{sc}(U \amalg V) \leqslant 2 n m$ if $|\Sigma|>1$ and $\operatorname{sc}(U ш V) \leqslant n m$ if $|\Sigma|=1$. Furthermore, for any $\Sigma$, there exist $U, V$ as above such that $n m \leqslant \operatorname{sc}(U \sqcup V)$.

2. In the worst case, $n$ states are sufficient and necessary for a DFA to recognize $\uparrow U$. Similarly for the downward closure and interior operations.

3. In the worst case, $n$ states are sufficient and necessary for a DFA to recognize the projection of $U$.

4. In the worst case, $n m$ states are sufficient and necessary for a DFA to recognize $U \cap V$ or $U \cup V$.

Proof. 1. If $|\Sigma|=1$, then the shuffle operation is the same as concatenation and the claim follows by the state complexity of concatenation on unary languages, see [YZS94]. Otherwise, with Theorem 4 .

$$
\begin{aligned}
\operatorname{sc}(U ш V) & \leqslant \prod_{l=1}^{k}\left(i_{l}+j_{l}+2 \operatorname{lcm}\left(p_{l}, q_{l}\right)-1\right) \\
& \leqslant \prod_{l=1}^{k} 2\left(i_{l}+p_{l}\right)\left(j_{l}+q_{l}\right)=2 \cdot \prod_{l=1}^{k}\left(i_{l}+p_{l}\right) \prod_{l=1}^{k}\left(j_{l}+q_{l}\right)=2 n m .
\end{aligned}
$$

Let $p, q>0$ be two coprime numbers and set $U=\bigsqcup_{j=1}^{k} a_{j}^{p-1}\left(a_{j}^{p}\right)^{*}$ and $V=$ $\bigsqcup_{j=1}^{k} a_{j}^{q-1}\left(a_{j}^{q}\right)^{*}$. By Lemma 10 both have minimal automata of productform and $\operatorname{sc}(U)=p^{k}$ and $\operatorname{sc}(V)=q^{k}$ Also, $\operatorname{sc}\left(a_{j}^{p-1}\left(a_{j}^{p}\right)^{*} \cdot a_{j}^{q-1}\left(a_{j}^{q}\right)^{*}\right)=p q$, see [YZS94, Lemma 5.1 and Fact 5.2]. Further,

$$
U ш V=\bigsqcup_{j=1}^{k}\left(a_{j}^{p-1}\left(a_{j}^{p}\right)^{*} \cdot a_{j}^{q-1}\left(a_{j}^{q}\right)^{*}\right) .
$$

So, with Lemma 10, the minimal automaton of $U \uplus V$ has product-form and $\operatorname{sc}(U \amalg V)=p^{k} \cdot q^{k}$.

2. This is a direct application of Theorem 5, as by assumption and Theorem 9 we have $n=\prod_{l=1}^{k}\left(i_{l}+p_{l}\right)$. For the lower bound, use $L=a^{n} \amalg(\Sigma \backslash\{a\})^{*}$. Then, the upward closure is $a^{n} a^{*} \amalg(\Sigma \backslash\{a\})^{*}$ and the downward closure is $\left\{\varepsilon, a, \ldots, a^{n}\right\} \amalg(\Sigma \backslash\{a\})^{*}$. 
3. Set $L=\uplus_{j=1}^{k} a_{j}^{*}=\Sigma^{*}$. Then, $\operatorname{sc}(L)=1$. Or, for any $n>1$, where we assume $\Sigma=\{a, b\}$ for notational simplicity, we have $L=a^{*} \amalg b^{n} b^{*}$, then $\operatorname{sc}(L)=n+1$ and $\operatorname{sc}\left(\pi_{\{b\}}(L)\right)=n+1$.

4. The stated bound is valid for union and intersection in general, see [YZS94, Theorem 4.3].

Let $p, q>0$ be two coprime numbers and set $U=\bigsqcup_{j=1}^{k} a_{j}^{p-1}\left(a_{j}^{p}\right)^{*}$ and $V=$ $\bigsqcup_{j=1}^{k} a_{j}^{q-1}\left(a_{j}^{q}\right)^{*}$. By Lemma 10 both have minimal automata of productform. Also, $\operatorname{sc}\left(a_{j}^{p-1}\left(a_{j}^{p}\right)^{*} \cap a_{j}^{q-1}\left(a_{j}^{q}\right)^{*}\right)=p q$ and

$$
U \cap V=\bigsqcup_{j=1}^{k}\left(a_{j}^{p-1}\left(a_{j}^{p}\right)^{*} \cap a_{j}^{q-1}\left(a_{j}^{q}\right)^{*}\right) .
$$

So, with Lemma 10 the minimal automaton of $U \cap V$ has product-form. As this property is closed under complementation by Theorem 11 the statement for union is implied.

This finishes the proof.

\section{Proofs for Section 4 (Partial Commutativity and Other Subclasses)}

Example 7. Let $\Sigma=\Sigma_{1} \cup \Sigma_{2}$ with $\Sigma_{1}=\{a, b\}$ and $\Sigma_{2}=\{c, d\}$. Then, in the language $U=\{a b c d, a c b d, c a b d, c a d b, c d a b\}$ the letters commute according to the partition $\Sigma=\Sigma_{1} \cup \Sigma_{2}$. On the contrary, in the language $V=\{a b c, b a c, c b a\}$ the letters do not commute according to the partition, as, for example, $a b c \in L$, but $c a b \notin L$.

Remark 6. Let $\Sigma=\Sigma_{1} \cup \ldots \cup \Sigma_{k}$ and $L \subseteq \Sigma^{*}$ be such that the letters in $L$ commute according to the partition. Then, for each $w \in \Sigma^{*}$,

$$
w \in L \Leftrightarrow \pi_{\Sigma_{1}}(w) \pi_{\Sigma_{2}}(w) \cdot \ldots \cdot \pi_{\Sigma_{k}}(w) \in L
$$

and $w \equiv_{L} \pi_{\Sigma_{1}}(w) \pi_{\Sigma_{2}}(w) \cdot \ldots \cdot \pi_{\Sigma_{k}}(w)$.

The next lemma will be needed when we relate the different subclasses in Subsection 4 .

Lemma 24. If $L \in \mathcal{L}_{\Sigma_{1}, \ldots, \Sigma_{k}}$ and $u \in \Sigma_{i}^{*}$, then $[u]_{\equiv_{L}} \cap \Sigma_{i}^{*} \subseteq[u]_{\equiv_{\pi_{\Sigma_{i}}(L)}} \cap \Sigma_{i}^{*}$.

Proof. Suppose $u, v \in \Sigma_{i}^{*}, i \in\{1, \ldots, k\}$, and $u \equiv_{L} v$. If $x \in \Sigma_{i}^{*}$ and $u x \in \pi_{\Sigma_{i}}(L)$, then $u x=\pi_{\Sigma_{i}}(w)$ for some $w \in L$. Set

$$
w^{\prime}=\pi_{\Sigma_{1}}(w) \cdots \pi_{\Sigma_{i-1}}(w) v x \pi_{\Sigma_{i+1}}(w) \cdots \pi_{\Sigma_{k}}(w) .
$$

Then, using that $\equiv_{L}$ is a right-congruence, $L \in \mathcal{L}_{\Sigma_{1}, \ldots, \Sigma_{k}}$ and Remark 6 ,

$$
\begin{aligned}
{[w]_{\equiv_{L}} } & =\left[\pi_{\Sigma_{1}}(w) \cdots \pi_{\Sigma_{i-1}}(w) u x \pi_{\Sigma_{i+1}}(w) \cdots \pi_{\Sigma_{k}}(w)\right]_{\equiv_{L}} \\
& =\left[u \pi_{\Sigma_{1}}(w) \cdots \pi_{\Sigma_{i-1}}(w) x \pi_{\Sigma_{i+1}}(w) \cdots \pi_{\Sigma_{k}}(w)\right]_{\equiv_{L}} \\
& =\left[v \pi_{\Sigma_{1}}(w) \cdots \pi_{\Sigma_{i-1}}(w) x \pi_{\Sigma_{i+1}}(w) \cdots \pi_{\Sigma_{k}}(w)\right]_{\equiv_{L}} \\
& =\left[\pi_{\Sigma_{1}}(w) \cdots \pi_{\Sigma_{i-1}}(w) v x \pi_{\Sigma_{i+1}}(w) \cdots \pi_{\Sigma_{k}}(w)\right]_{\equiv_{L}}
\end{aligned}
$$

So, $w^{\prime} \equiv_{L} w$, and hence, $w^{\prime} \in L$. Also, $\pi_{\Sigma_{i}}\left(w^{\prime}\right)=v x$. So, $v x \in \pi_{\Sigma_{i}}(L)$. 
Remark 7. Let $\Sigma=\Sigma_{1} \cup \ldots \cup \Sigma_{k}$ be a partition, $u_{i}, v_{i} \in \Sigma^{*}, i \in\{1, \ldots, k\}$, and $w \in \Sigma^{*}$. Then, for the canonical automaton $\mathcal{C}_{L, \Sigma_{1}, \ldots, \Sigma_{k}}$, we have

$$
\begin{aligned}
\delta\left(\left(\left[u_{1}\right]_{\equiv_{L}}, \ldots,\left[u_{k}\right]_{\equiv_{L}}\right), w\right)=\left(\left[v_{1}\right]_{\equiv_{L}}, \ldots,\right. & {\left.\left[v_{k}\right]_{\equiv_{L}}\right) } \\
& \Leftrightarrow \forall i \in\{1, \ldots k\}: u_{i} \pi_{\Sigma_{i}}(w) \equiv_{L} v_{i} .
\end{aligned}
$$
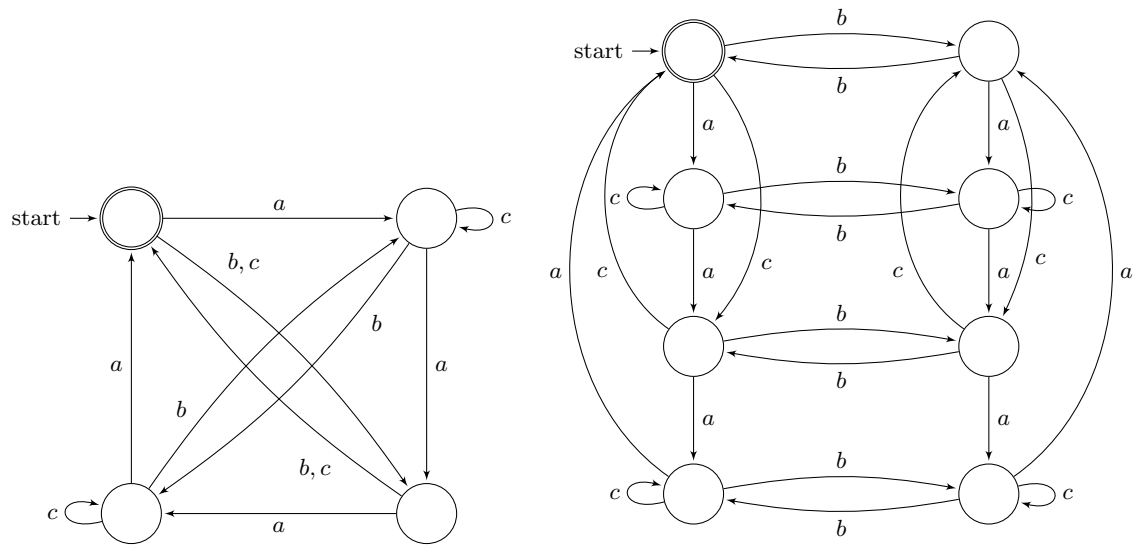

Fig. 4. On the left an automaton recognizing a language $L \in \mathcal{L}_{\{a, c\},\{b\}}$ and on the right the automaton $\mathcal{C}_{L,\{a, c,\},\{b\}}$.

Example 8. Please see Figure 4 for an automaton that recognizes a language in $\mathcal{L}_{\{a, c\},\{b\}}$ and the canonical automaton derived from it. That the recognized language is indeed in $\mathcal{L}_{\{a, c\},\{b\}}$ can be seen by noting that for each state $q \in Q$ we have $\delta(q, a b)=\delta(q, b a)$ and $\delta(q, c b)=\delta(q, b c)$.

Example 9. Let $\Sigma=\{a, b, c\}$ and $L=\Sigma^{*} a c^{*} b \Sigma^{*}$. Then $L \in \mathcal{L}_{\{a, b\},\{c\}}$. The minimal automaton of $L$ has a single final state and is isomorphic to $\mathcal{C}_{L,\{a, b\},\{c\}}$, as $[\varepsilon]_{\equiv_{L}}=\left[c^{n}\right]_{\equiv_{L}}$ for each $n \geqslant 0$. The language $L \cap \Sigma^{*} c \Sigma^{*}$ also has the property that the minimal automaton is isomorphic to $\mathcal{C}_{L \cap \Sigma^{*} c \Sigma^{*},\{a, b\},\{c\}}$.

Theorem 14. Let $L \subseteq \Sigma^{*}$ and $\Sigma=\Sigma_{1} \cup \ldots \cup \Sigma_{k}$ be a partition. Then,

1. $L \subseteq L\left(\mathcal{C}_{L, \Sigma_{1}, \ldots, \Sigma_{k}}\right)$ and $L\left(\mathcal{C}_{L, \Sigma_{1}, \ldots, \Sigma_{k}}\right) \in \mathcal{L}_{\Sigma_{1}, \ldots, \Sigma_{k}}$.

2. $L=L\left(\mathcal{C}_{L}, \Sigma_{1}, \ldots, \Sigma_{k}\right) \Leftrightarrow L \in \mathcal{L}_{\Sigma_{1}, \ldots, \Sigma_{k}}$.

3. Let $L \in \mathcal{L}_{\Sigma_{1}, \ldots, \Sigma_{k}}$. Then $L$ is regular if and only if $\mathcal{C}_{L, \Sigma_{1}, \ldots, \Sigma_{k}}$ is finite.

Proof. 1. Let $\mathcal{C}_{L, \Sigma_{1}, \ldots, \Sigma_{k}}=\left(\Sigma, S_{1} \times \ldots \times S_{k}, \delta, s_{0}, F\right)$ be the canonical automaton for $L$. For each $w \in \Sigma^{*}$, we have

$$
\delta\left(s_{0}, w\right)=\left(\left[\pi_{\Sigma_{1}}(w)\right]_{\equiv_{L}}, \ldots,\left[\pi_{\Sigma_{k}}(w)\right]_{\equiv_{L}}\right) .
$$

If $w \in L$, then, by Definition 13, we find $\delta\left(s_{0}, w\right) \in F$. So, $L \subseteq L\left(\mathcal{C}_{L, \Sigma_{1}, \ldots, \Sigma_{K}}\right)$. By Definition 13. for each $a \in \Sigma_{i}, b \in \Sigma_{j}, i \neq j, i, j \in\{1, \ldots, k\}$ and state 
$s \in S_{1} \times \ldots \times S_{k}$, we have $\delta(s, a b)=\delta(s, b a)$. So, we conclude $L\left(\mathcal{C}_{L, \Sigma_{1}, \ldots, \Sigma_{k}}\right) \in$ $\mathcal{L}_{\Sigma_{1}, \ldots, \Sigma_{k}}$.

2. If $L=L\left(\mathcal{C}_{L, \Sigma_{1}, \ldots, \Sigma_{k}}\right)$, then, by the previous item, $L \in \mathcal{L}_{\Sigma_{1}, \ldots, \Sigma_{k}}$. Now, suppose $L \in \mathcal{L}_{\Sigma_{1}, \ldots, \Sigma_{k}}$. By the previous item, we only have to establish $L\left(\mathcal{C}_{L, \Sigma_{1}, \ldots, \Sigma_{k}}\right) \subseteq L$. So, suppose $w \in L\left(\mathcal{C}_{L, \Sigma_{1}, \ldots, \Sigma_{k}}\right)$. By Definition 13, there exists $u \in L$ such that $\pi_{\Sigma_{i}}(w) \equiv_{L} \pi_{\Sigma_{i}}(u)$ for $i \in\{1, \ldots, k\}$. As the letters in $L$ commute according to the partition, we have, by Remark [6] as $u \in L$,

$$
\pi_{\Sigma_{1}}(u) \pi_{\Sigma_{2}}(u) \pi_{\Sigma_{3}}(u) \cdots \pi_{\Sigma_{k}}(u) \in L .
$$

So, using $\pi_{\Sigma_{1}}(w) \equiv_{L} \pi_{\Sigma_{1}}(u)$, we find

$$
\pi_{\Sigma_{1}}(w) \pi_{\Sigma_{2}}(u) \pi_{\Sigma_{3}}(u) \cdots \pi_{\Sigma_{k}}(u) \in L .
$$

Using that the letters in $L$ commute according to the partition again, we find, with the previous equation,

$$
\pi_{\Sigma_{2}}(u) \pi_{\Sigma_{1}}(w) \pi_{\Sigma_{3}}(u) \cdots \pi_{\Sigma_{k}}(u) \in L .
$$

And then, using $\pi_{\Sigma_{2}}(w) \equiv_{L} \pi_{\Sigma_{2}}(u)$,

$$
\pi_{\Sigma_{2}}(w) \pi_{\Sigma_{1}}(w) \pi_{\Sigma_{3}}(u) \cdots \pi_{\Sigma_{k}}(u) \in L .
$$

Continuing in this manner, and reordering the result, we get

$$
\pi_{\Sigma_{1}}(w) \pi_{\Sigma_{2}}(w) \pi_{\Sigma_{3}}(w) \cdots \pi_{\Sigma_{k}}(w) \in L
$$

So, as the letters in $L$ commute according to the partition, $w \in L$. Hence $L\left(\mathcal{C}_{L, \Sigma_{1}, \ldots, \Sigma_{k}}\right) \subseteq L$.

3. As $\mathcal{C}_{L, \Sigma_{1}, \ldots \Sigma_{k}}$ is defined with the Nerode right-congruence classes, if $L$ is regular, it must be a finite automaton. If the automaton is finite and $L \in$ $\mathcal{L}_{\Sigma_{1}, \ldots, \Sigma_{k}}$, then, by the previous item, $L=L\left(\mathcal{C}_{L, \Sigma_{1}, \ldots, \Sigma_{k}}\right)$ and $L$ is regular. So, we have established all statements in the theorem.

Proposition 16. Let $L \in \mathcal{L}_{\Sigma_{1}, \ldots, \Sigma_{k}}$. Then, for $i \in\{1, \ldots, k\}, \pi_{\Sigma_{i}}(L)=L\left(\mathcal{C}_{L, \Sigma_{i}}\right)$.

Proof. Let $\mathcal{C}_{L, \Sigma_{i}}=\left(\Sigma, S_{i}, \delta_{i},[\varepsilon]_{\equiv_{L}}, F_{i}\right)$. Suppose $u \in \pi_{\Sigma_{i}}(L)$. Then, there exists $w \in L$ such that $u=\pi_{\Sigma_{i}}(w)$. So, $[u]_{\equiv_{L}} \in F_{i}$. Hence, $u \in L\left(\mathcal{C}_{L, \Sigma_{i}}\right)$.

Conversely, if $u \in L\left(\mathcal{C}_{L, \Sigma_{i}}\right) \subseteq \Sigma_{i}^{*}$, then $u \equiv_{L} \pi_{\Sigma_{i}}(w)$ for some $w \in L$. We have $\pi_{\Sigma_{i}}(w) \prod_{j=1, j \neq i}^{k} \pi_{\Sigma_{j}}(w) \in L$. So, $u \prod_{j=1, j \neq i}^{k} \pi_{\Sigma_{j}}(w) \in L$, which gives $u \in \pi_{\Sigma_{j}}(L)$.

Proposition 18, Let $\Sigma=\Sigma_{1} \cup \ldots \cup \Sigma_{k}$ be a partition. For each $i \in\{1,2,3,4\}$ we have $\mathcal{L}_{i} \subseteq \mathcal{L}_{\Sigma_{1}, \ldots, \Sigma_{k}}$.

Proof. If $i \in\{1,3,4\}$, by Theorem [14, we have $\mathcal{L}_{i} \subseteq \mathcal{L}_{\Sigma_{1}, \ldots, \Sigma_{k}}$. By the definition of the shuffle product, $\mathcal{L}_{2} \subseteq \mathcal{L}_{\Sigma_{1}, \ldots, \Sigma_{k}}$.

\footnotetext{
${ }^{2}$ Set, for $u_{1}, \ldots, u_{n} \in \Sigma^{*}, \prod_{i=1}^{n} u_{i}=u_{1} \cdot \ldots \cdot u_{n}$.
} 
Proposition 19, Let $L \in \mathcal{L}_{\Sigma_{1}, \ldots, \Sigma_{k}}$ and $\mathcal{C}_{L, \Sigma_{1}, \ldots, \Sigma_{k}}=\left(\Sigma, S_{1} \times \ldots \times S_{k}, \delta, s_{0}, F\right)$. Then, for all $s \in S_{1} \times \ldots \times S_{k},\left\{w \in \Sigma^{*} \mid \delta\left(s_{0}, w\right)=s\right\} \in \mathcal{L}_{1}$.

Proof. Let $s=\left(s_{1}, \ldots, s_{k}\right) \in S_{1} \times \ldots \times S_{k}$. Set $U=\left\{w \in \Sigma^{*} \mid \delta\left(s_{0}, w\right)=s\right\}$. By construction of $\mathcal{C}_{L, \Sigma_{1}, \ldots, \Sigma_{k}}$, we have $U \in \mathcal{L}_{\Sigma_{1}, \ldots, \Sigma_{k}}$. So, by Theorem 14, $U=L\left(\mathcal{C}_{U, \Sigma_{1}, \ldots, \Sigma_{k}}\right)$, where $\mathcal{C}_{U, \Sigma_{1}, \ldots, \Sigma_{k}}$ is the canonical automaton for $U$. Let

$$
E=\left\{\left(\left[\pi_{\Sigma_{1}}(u)\right]_{\equiv_{U}}, \ldots,\left[\pi_{\Sigma_{k}}(u)\right]_{\equiv_{U}}\right) \mid u \in U\right\}
$$

be the final states of $\mathcal{C}_{U, \Sigma_{1}, \ldots, \Sigma_{k}}$. We have to show $|E|=1$. Let $u_{i} \in \Sigma_{i}^{*}$ be such that $s_{i}=\left[u_{i}\right]_{\equiv_{L}}$ for $i \in\{1, \ldots, k\}$.

Claim: For all $v \in \Sigma_{i}^{*}$ and $u \in U$ we have

$$
v \equiv_{U} \pi_{\Sigma_{i}}(u) \Leftrightarrow v \equiv_{L} u_{i}
$$

Proof of the Claim: We show two separate statements that, taken together, imply our claim.

1. For all $i \in\{1, \ldots, k\}$ and $u \in U,\left[\pi_{\Sigma_{i}}(u)\right]_{\equiv_{U}} \cap \Sigma_{i}^{*} \subseteq\left[u_{i}\right]_{\equiv_{L}}$.

Fix $i \in\{1, \ldots, k\}$. Suppose $x \in \Sigma_{i}^{*}$ such that $x \equiv_{U} \pi_{\Sigma_{i}}(u)$. Then, as

$$
\pi_{\Sigma_{i}}(u) \pi_{\Sigma_{1}}(u) \cdots \pi_{\Sigma_{i-1}}(u) \pi_{\Sigma_{i+1}}(u) \cdots \pi_{\Sigma_{k}}(u) \in U
$$

we find, by the definition of the Nerode right-congruence,

$$
x \pi_{\Sigma_{1}}(u) \cdots \pi_{\Sigma_{i-1}}(u) \pi_{\Sigma_{i+1}}(u) \cdots \pi_{\Sigma_{k}}(u) \in U .
$$

Hence, $\delta\left(s_{0}, x \pi_{\Sigma_{1}}(u) \cdots \pi_{\Sigma_{i-1}}(u) \pi_{\Sigma_{i+1}}(u) \cdots \pi_{\Sigma_{k}}(u)\right)=s$. By Re$\operatorname{mark} 7$ as $x \in \Sigma_{i}^{*}$, we find $x \equiv_{L} u_{i}$.

2. For all $i \in\{1, \ldots, k\}$, if $x, y \in\left[u_{i}\right]_{\equiv_{L}} \cap \Sigma_{i}^{*}$, then $x \equiv_{U} y$.

Fix $i \in\{1, \ldots, k\}$. Assume there exist $x, y \in \Sigma_{i}^{*}$ such that $x \neq_{U} y$, but $x, y \in\left[u_{i}\right]_{\equiv_{L}}$. Then, without loss of generality, there exists $z \in \Sigma^{*}$ such that

$$
x z \in U, \quad y z \notin U .
$$

But then, for all $j \in\{1, \ldots, k\}, \pi_{\Sigma_{j}}(x z) \equiv_{L} u_{j}$. As $x, y \in \Sigma_{i}^{*}$, for $j \neq i$, we also have $\pi_{\Sigma_{j}}(y z) \equiv_{L} u_{j}$. So, for $y z \notin U$ to hold true, we must have $\pi_{\Sigma_{i}}(y z) \equiv_{L} u_{i}$. However, by assumption $x, y \in\left[u_{i}\right]_{\equiv_{L}}$, so

$$
\pi_{\Sigma_{i}}(y z)=y \pi_{\Sigma_{i}}(z) \equiv_{L} x \pi_{\Sigma_{i}}(z)=\pi_{\Sigma_{i}}(x z) \equiv_{L} u_{i},
$$

which implies $\pi_{\Sigma_{i}}(y z) \equiv_{L} u_{i}$. So, $y z \notin U$ is not possible. Hence, all words from $\Sigma_{i}^{*}$ in $\left[u_{i}\right]_{\equiv_{L}}$ must be equivalent for $\equiv_{U}$.

Combining the first and second part gives, for each $i \in\{1, \ldots, k\}$ and $u \in U,\left[\pi_{\Sigma_{i}}(u)\right]_{\equiv_{U}} \cap \Sigma_{i}^{*}=\left[u_{i}\right]_{\equiv_{L}} \cap \Sigma_{i}^{*}$. [End, Proof of the Claim $]$ 
Now, choose $u, u^{\prime} \in U$ and $i \in\{1, \ldots, k\}$. Then, by the above claim, we find

$$
\pi_{\Sigma_{i}}(u) \equiv_{L} u_{i} \text { and } \pi_{\Sigma_{i}}\left(u^{\prime}\right) \equiv_{L} u_{i} .
$$

Using the above claim, with $v=\pi_{\Sigma_{i}}\left(u^{\prime}\right)$, we can then deduce $\pi_{\Sigma_{i}}\left(u^{\prime}\right) \equiv_{U} \pi_{\Sigma_{i}}(u)$. So, for each $u, u^{\prime} \in U$, we have

$$
\left(\left[\pi_{\Sigma_{1}}(u)\right]_{\equiv_{U}}, \ldots,\left[\pi_{\Sigma_{k}}(u)\right]_{\equiv_{U}}\right)=\left(\left[\pi_{\Sigma_{1}}\left(u^{\prime}\right)\right]_{\equiv_{U}}, \ldots,\left[\pi_{\Sigma_{k}}\left(u^{\prime}\right)\right]_{\equiv_{U}}\right),
$$

which implies $|E|=1$.

Theorem [20. Let $L \in \mathcal{L}_{\Sigma_{1}, \ldots, \Sigma_{k}}$. Then,

1. $L \in \mathcal{L}_{2}$ if and only if, for each $w \in \Sigma^{*}$, the following is true:

$$
w \in L \Leftrightarrow \forall i \in\{1, \ldots, k\}: \pi_{\Sigma_{i}}(w) \in \pi_{\Sigma_{i}}(L)
$$

2. $L \in \mathcal{L}_{3}$ if and only if, for all $i \in\{1, \ldots, k\}$ and $u \in \Sigma_{i}^{*}$, we have

$$
[u]_{\equiv_{L}} \cap \Sigma_{i}^{*}=[u]_{\equiv_{\pi_{\Sigma_{i}}(L)}} \cap \Sigma_{i}^{*}
$$

3. $L \in \mathcal{L}_{4}$ if and only if, for each $u, v \in \Sigma^{*}$,

$$
u \equiv_{L} v \Leftrightarrow \forall i \in\{1, \ldots, k\}: \pi_{\Sigma_{i}}(u) \equiv_{L} \pi_{\Sigma_{i}}(v) .
$$

Proof. The separate claims are stated in Proposition 25] Proposition 26] and Proposition 27

Note that, for each $L \subseteq \Sigma^{*}$,

$$
L \subseteq \bigsqcup_{i=1}^{k} \pi_{\Sigma_{i}}(L)
$$

Proposition 25. Let $L \in \mathcal{L}_{\Sigma_{1}, \ldots, \Sigma_{k}}$. Then, $L \in \mathcal{L}_{2}$ if and only if, for each $w \in \Sigma^{*}$, the following is true:

$$
w \in L \Leftrightarrow \forall i \in\{1, \ldots, k\}: \pi_{\Sigma_{i}}(w) \in \pi_{\Sigma_{i}}(L) .
$$

Proof. 1. Suppose $L=\bigsqcup_{i=1}^{k} \pi_{\Sigma_{i}}(L)$. We show that the equivalence holds true. If $w \in L$, then, for each $i \in\{1, \ldots, k\}, \pi_{\Sigma_{i}}(w) \in \pi_{\Sigma_{i}}(L)$. Conversely, let $w \in \Sigma^{*}$ and assume, for each $i \in\{1, \ldots, k\}$, we have $\pi_{\Sigma_{i}}(w) \in \pi_{i}(L)$. Then, $w \in \bigsqcup_{i=1}^{k} \pi_{\Sigma_{i}}(w) \subseteq \bigsqcup_{i=1}^{k} \pi_{\Sigma_{i}}(L)=L$.

2. Now, suppose, for each $w \in \Sigma^{*}$, we have

$$
w \in L \Leftrightarrow \forall i \in\{1, \ldots, k\}: \pi_{\Sigma_{i}}(w) \in \pi_{i}(L) .
$$

By Equation (7), $L \subseteq \bigsqcup_{i=1}^{k} \pi_{\Sigma_{i}}(L)$. So, assume $w \in \bigsqcup_{i=1}^{k} \pi_{\Sigma_{i}}(L)$. Fix $j \in$ $\{1, \ldots, k\}$. Then, as $\Sigma_{1}, \ldots, \Sigma_{k}$ are pairwise disjoint and so $\pi_{\Sigma_{i}}\left(\pi_{\Sigma_{j}}(L)\right)=$ $\{\varepsilon\}$ for $i \neq j$,

$$
\pi_{\Sigma_{j}}(w) \in \pi_{\Sigma_{j}}\left(\bigsqcup_{i=1}^{k} \pi_{\Sigma_{i}}(L)\right)=\bigsqcup_{i=1}^{k} \pi_{\Sigma_{j}}\left(\pi_{\Sigma_{i}}(L)\right)=\pi_{\Sigma_{j}}(L) .
$$

Hence, by applying the equation, we find $w \in L$. So, $\bigsqcup_{i=1}^{k} \pi_{\Sigma_{i}}(L) \subseteq L$. So, we have shown the equivalence of both conditions. 
Proposition 26. Let $L \in \mathcal{L}_{\Sigma_{1}, \ldots, \Sigma_{k}}$. Then, $L \in \mathcal{L}_{3}$ if and only if, for each $i \in\{1, \ldots, k\}$ and $u \in \Sigma_{i}^{*}$, we have $[u]_{\equiv_{L}} \cap \Sigma_{i}^{*}=[u]_{\equiv_{\Sigma_{\Sigma_{i}}(L)}} \cap \Sigma_{i}^{*}$.

Proof. The map $\varphi:\left\{[u]_{\equiv_{L}} \cap \Sigma_{i}^{*} \mid u \in \Sigma_{i}^{*}\right\} \rightarrow\left\{[u]_{\pi_{\Sigma_{i}}(L)} \cap \Sigma_{i}^{*} \mid u \in \Sigma_{i}^{*}\right\}$ given by

$$
\varphi\left([u]_{\equiv_{L}} \cap \Sigma_{i}^{*}\right)=[u]_{\equiv_{\pi_{\Sigma_{i}}(L)}} \cap \Sigma_{i}^{*}
$$

is well-defined by Lemma 24 . Also, the non-empty sets $[u]_{\equiv_{L}} \cap \Sigma_{i}^{*}$ partition $\Sigma_{i}^{*}$ as a right-congruence over $\Sigma_{i}^{*}$. Moreover, the non-empty right-congruence classes of the form $[u]_{\equiv_{L}} \cap \Sigma_{i}^{*}$ are precisely the states of $\mathcal{C}_{L, \Sigma_{i}}$. So, $\varphi$ induces a surjective homomorphism from $\mathcal{C}_{L, \Sigma_{i}}$ onto $\mathcal{A}_{\pi_{\Sigma_{i}}(L)}$. This yields the stated equivalence.

Proposition 27. Let $L \in \mathcal{L}_{\Sigma_{1}, \ldots, \Sigma_{k}}$. Then, $L \in \mathcal{L}_{4}$ if and only if, for each $u, v \in \Sigma^{*}$,

$$
u \equiv_{L} v \Leftrightarrow \forall i \in\{1, \ldots, k\}: \pi_{\Sigma_{i}}(u) \equiv_{L} \pi_{\Sigma_{i}}(v)
$$

Proof. First, we show that the implication from right to left is always true. Hence, we only have to argue that if the other implication is true, this is equivalent to $L \in \mathcal{L}_{4}$.

Claim: Let $L \in \mathcal{L}_{\Sigma_{1}, \ldots, \Sigma_{k}}$ and $u, v \in \Sigma^{*}$. Then,

$$
\forall i \in\{1, \ldots, k\}: \pi_{\Sigma_{i}}(u) \equiv_{L} \pi_{\Sigma_{i}}(v) \Rightarrow u \equiv_{L} v .
$$

Proof of the Claim: Suppose

$$
\forall i \in\{1, \ldots, k\}: \pi_{\Sigma_{i}}(u) \equiv_{L} \pi_{\Sigma_{i}}(v) .
$$

Then, using $\pi_{\Sigma_{1}}(u) \equiv_{L} \pi_{\Sigma_{1}}(v)$, at it is a right-congruence, we find

$$
\pi_{\Sigma_{1}}(u) \pi_{\Sigma_{2}}(v) \cdots \pi_{\Sigma_{k}}(v) \equiv_{L} \pi_{\Sigma_{1}}(v) \pi_{\Sigma_{2}}(v) \cdots \pi_{\Sigma_{k}}(v) .
$$

As $L \in \mathcal{L}_{\Sigma_{1}, \ldots, \Sigma_{k}}$, we have

$$
\pi_{\Sigma_{1}}(u) \pi_{\Sigma_{2}}(v) \pi_{\Sigma_{3}}(v) \cdots \pi_{\Sigma_{k}}(v) \equiv_{L} \pi_{\Sigma_{2}}(v) \pi_{\Sigma_{1}}(u) \pi_{\Sigma_{3}}(v) \cdots \pi_{\Sigma_{k}}(v) .
$$

Then, using $\pi_{\Sigma_{2}}(u) \equiv_{L} \pi_{\Sigma_{2}}(v)$,

$$
\pi_{\Sigma_{2}}(u) \pi_{\Sigma_{1}}(u) \pi_{\Sigma_{3}}(v) \cdots \pi_{\Sigma_{k}}(v) \equiv_{L} \pi_{\Sigma_{2}}(v) \pi_{\Sigma_{1}}(u) \pi_{\Sigma_{3}}(v) \cdots \pi_{\Sigma_{k}}(v) .
$$

Hence, up to now,

$$
\pi_{\Sigma_{2}}(u) \pi_{\Sigma_{1}}(u) \pi_{\Sigma_{3}}(v) \cdots \pi_{\Sigma_{k}}(v) \equiv_{L} \pi_{\Sigma_{2}}(v) \pi_{\Sigma_{1}}(v) \pi_{\Sigma_{3}}(v) \cdots \pi_{\Sigma_{k}}(v) .
$$

Continuing similarly, we can show that

$$
\pi_{\Sigma_{2}}(u) \cdots \pi_{\Sigma_{k}}(u) \equiv_{L} \pi_{\Sigma_{2}}(u) \cdots \pi_{\Sigma_{k}}(u) .
$$

Sd 3 , by Remark [6 $u \equiv_{L} v$. [End, Proof of the Claim]

\footnotetext{
${ }^{3}$ Alternatively, we can note that by commutativity, the Nerode right-congruence is also a left-congruence. Hence, it is the syntactic congruence, giving a natural composition operation on the equivalence classes. Then, by Remark 6

$$
[u]_{\equiv_{L}}=\left[\pi_{\Sigma_{1}}(u) \cdots \pi_{\Sigma_{k}}(u)\right]_{\equiv_{L}}=\left[\pi_{\Sigma_{1}}(u)\right]_{\equiv_{L}} \cdots\left[\pi_{\Sigma_{k}}(u)\right]_{\equiv_{L}},
$$

which also gives that, if for all $i \in\{1, \ldots, k\}$ we have $\pi_{\Sigma_{i}}(u) \equiv_{L} \pi_{\Sigma_{i}}(v)$, then $u \equiv_{L} v$.
} 
Therefore, as

$$
\forall i \in\{1, \ldots, k\}: \pi_{\Sigma_{i}}(u) \equiv_{L} \pi_{\Sigma_{i}}(v) \Rightarrow u \equiv_{L} v,
$$

the map

$$
\left(\left[\pi_{\Sigma_{1}}\left(u_{1}\right)\right]_{\equiv_{L}}, \ldots,\left[\pi_{\Sigma_{k}}\left(u_{k}\right)\right]_{\equiv_{L}}\right) \mapsto\left[\pi_{\Sigma_{1}}\left(u_{1}\right) \cdots \pi_{\Sigma_{k}}\left(u_{k}\right)\right]_{\equiv_{L}} .
$$

for $u_{1}, \ldots, u_{k} \in \Sigma^{*}$ is well-defined. For, suppose $v_{1}, \ldots, v_{k} \in \Sigma^{*}$ such that

$$
\forall i \in\{1, \ldots, k\}: \pi_{\Sigma_{i}}\left(u_{i}\right) \equiv_{L} \pi_{\Sigma_{i}}\left(v_{i}\right) .
$$

Set $u=\pi_{\Sigma_{1}}\left(u_{1}\right) \cdots \pi_{\Sigma_{k}}\left(u_{k}\right)$ and $v=\pi_{\Sigma_{1}}\left(v_{1}\right) \cdots \pi_{\Sigma_{k}}\left(v_{k}\right)$. Then, for all $i \in$ $\{1, \ldots, k\}$, we have $\pi_{\Sigma_{i}}(u)=\pi_{\Sigma_{i}}\left(u_{i}\right)$ and $\pi_{\Sigma_{i}}(v)=\pi_{\Sigma_{i}}\left(v_{i}\right)$, which implies $\pi_{\Sigma_{i}}(u) \equiv_{L} \pi_{\Sigma_{i}}(v)$ and we find that $u \equiv_{L} v$. Also, as $\left(\left[\pi_{\Sigma_{1}}(u)\right]_{\equiv_{L}}, \ldots,\left[\pi_{\Sigma_{k}}(u)\right]\right)$ gets mapped to $\left[\pi_{\Sigma_{1}}(u) \cdots \pi_{\Sigma_{k}}(u)\right]_{\equiv_{L}}=[u]_{\equiv_{L}}$, the map is surjective. By commutativity, we can also easily show that it is an automaton homomorphism.

So, if $L \in \mathcal{L}_{4}$, then $\mathcal{C}_{L, \Sigma_{1}, \ldots, \Sigma_{k}}$ and $\mathcal{A}_{L}$ have the same number of states. Hence, the above given surjective map is actually bijective. So,

$$
\left[\pi_{\Sigma_{1}}\left(u_{1}\right) \cdots \pi_{\Sigma_{k}}\left(u_{k}\right)\right]_{\equiv_{L}}=\left[\pi_{\Sigma_{1}}\left(v_{1}\right) \cdots \pi_{\Sigma_{k}}\left(v_{k}\right)\right]_{\equiv_{L}}
$$

implies

$$
\left(\left[\pi_{\Sigma_{1}}\left(u_{1}\right)\right]_{\equiv_{L}}, \ldots,\left[\pi_{\Sigma_{k}}\left(u_{k}\right)\right]_{\equiv_{L}}\right)=\left(\left[\pi_{\Sigma_{1}}\left(v_{1}\right)\right]_{\equiv_{L}}, \ldots,\left[\pi_{\Sigma_{k}}\left(v_{k}\right)\right]_{\equiv_{L}}\right) .
$$

In particular, if $u \equiv_{L} v$, then, by Remark 6 , we have $\left[\pi_{\Sigma_{1}}(u) \cdots \pi_{\Sigma_{k}}(u)\right]_{\equiv_{L}}=$ $\left[\pi_{\Sigma_{1}}(v) \cdots \pi_{\Sigma_{k}}(v)\right]_{\equiv_{L}}$, which gives

$$
\left(\left[\pi_{\Sigma_{1}}(u)\right]_{\equiv_{L}}, \ldots,\left[\pi_{\Sigma_{k}}(u)\right]_{\equiv_{L}}\right)=\left(\left[\pi_{\Sigma_{1}}(v)\right]_{\equiv_{L}}, \ldots,\left[\pi_{\Sigma_{k}}(v)\right]_{\equiv_{L}}\right)
$$

and we have the implication

$$
u \equiv_{L} v \Rightarrow \forall i \in\{1, \ldots, k\}: \pi_{\Sigma_{i}}(u) \equiv_{L} \pi_{\Sigma_{i}}(v) .
$$

Conversely, suppose Equation (8) holds true. Then, the map

$$
[u]_{\equiv_{L}} \mapsto\left(\left[\pi_{\Sigma_{1}}(u)\right]_{\equiv_{L}}, \ldots,\left[\pi_{\Sigma_{k}}(u)\right]_{\equiv_{L}}\right)
$$

is well-defined. As, for $u_{1}, \ldots, u_{k} \in \Sigma^{*}, u=\pi_{\Sigma_{1}}\left(u_{1}\right) \cdots \pi_{\Sigma_{k}}\left(u_{k}\right)$ gets mapped to $\left(\left[\pi_{\Sigma_{1}}\left(u_{1}\right)\right]_{\equiv_{L}}, \ldots,\left[\pi_{\Sigma_{k}}\left(u_{k}\right)\right]_{\equiv_{L}}\right)$, it is also surjective. So, by finitenes: 4 , it is a bijection between $\mathcal{A}_{L}$ and $\mathcal{C}_{L, \Sigma_{1}, \ldots, \Sigma_{k}}$.

${ }^{4}$ Actually, we do not need to use finitess, and can show it directly by noting that the given map is a two-sided inverse to the map

$$
\left(\left[\pi_{\Sigma_{1}}\left(u_{1}\right)\right]_{\equiv_{L}}, \ldots,\left[\pi_{\Sigma_{k}}\left(u_{k}\right)\right]_{\equiv_{L}}\right) \mapsto\left[\pi_{\Sigma_{1}}\left(u_{1}\right) \cdots \pi_{\Sigma_{k}}\left(u_{k}\right)\right]_{\equiv_{L}} .
$$


Remark 8. Note that the condition in Proposition 26 is stated only for words from $\Sigma_{i}^{*}, i \in\{1, \ldots, k\}$. For example, stipulating the equation

$$
[u]_{\equiv_{L}} \cap \Sigma_{i}^{*}=\left[\pi_{\Sigma_{i}}(u)\right]_{\equiv_{\pi_{\Sigma_{i}}(L)}} \cap \Sigma_{i}^{*}
$$

for all $u \in \Sigma^{*}$ is a strong demand. It implies, for $a \in \Sigma_{i}, b \in \Sigma_{j}, i \neq j$, $[a b]_{\equiv_{L}}=[a]_{\equiv_{L}}=[b]_{\equiv_{L}}$, as

$$
\begin{aligned}
& {[a b]_{\equiv_{L}} \cap \Sigma_{i}^{*}=[a]_{\equiv_{\pi_{\pi_{\Sigma_{i}}(L)}}} \cap \Sigma_{i}^{*} ;} \\
& {[a b]_{\equiv_{L}} \cap \Sigma_{j}^{*}=[b]_{\equiv_{\pi_{\pi_{\Sigma_{j}}(L)}}} \cap \Sigma_{j}^{*},}
\end{aligned}
$$

which gives $a, b \in[a b]_{\equiv_{L}}$.

Remark 9. Let $L \subseteq \Sigma^{*}$. In several statements, we have intersected the equivalence classes for $\pi_{\Sigma_{i}}(L), i \in\{1, \ldots, k\}$, with $\Sigma_{i}^{*}$. Equivalently, we could also say that we only consider the equivalence over the smaller alphabet $\Sigma_{i}^{*}$. More generally, if $\Gamma \subseteq \Sigma$ and $U \subseteq \Gamma^{*}$, then, $u, v \in \Gamma^{*}$ are equivalent for $\equiv_{U}$ over the alphabet $\Gamma$ if and only if they are equivalent for $\equiv_{U}$ over $\Sigma$. Also, over the larger alphabet, all words using symbols not in $\Gamma$ are equivalent.

Remark 10. The condition in Proposition 26 does not imply that the language is in $\mathcal{L}_{\Sigma_{1}, \ldots, \Sigma_{k}}$. Let $L=\{a b, b\}$. Then, $\pi_{\{a\}}(L)=\{a\}, \pi_{\{b\}}(L)=\{b\}$ and, for each $n, m \geqslant 0,\left[a^{n}\right]_{\equiv_{L}} \cap a^{*}=\left[a^{n}\right]_{\equiv_{\{a\}}}$ and $\left[b^{m}\right]_{\equiv_{L}} \cap b^{*}=\left[b^{m}\right]_{\equiv_{\{b\}}}$. However, $L \notin \mathcal{L}_{\{a\},\{b\}}$.

Example 10. For the language $L=\left(a(a a a)^{*} \cup a a(a a a)^{*}\right) \amalg b=a(a a a)^{*} \amalg b \cup$ $a a(a a a)^{*} \amalg b$ the minimal commutative automaton has more than a single final state, but $L=\pi_{1}(L) \amalg \pi_{2}(L)$.

Proposition 28. Let $L \in \mathcal{L}_{\Sigma_{1}, \ldots, \Sigma_{k}}$. Then, the following statements are equivalent:

1. $L=\bigsqcup_{i=1}^{k} \pi_{\Sigma_{i}}(L)$;

2. in the canonical automaton $\mathcal{C}_{L, \Sigma_{1}, \ldots, \Sigma_{k}}=\left(\Sigma, S_{1} \times \ldots \times S_{k}, \delta, s_{0}, F\right)$ we can write $F=F_{1} \times \ldots \times F_{k}$ with $F_{i} \subseteq S_{i}$.

Proof. 1. Suppose $L=\bigsqcup_{i=1}^{k} \pi_{\Sigma_{i}}(L)$. For the final state set $F$ of the canonical automaton, we will show $F=F_{1} \times \ldots \times F_{k}$.

First, note that, by Definition [13, we find $F \subseteq F_{1} \times \ldots \times F_{k}$. For the converse inclusion, let $u_{1}, \ldots, u_{k} \in L$, and consider the tuple

$$
\left(\left[\pi_{\Sigma_{1}}\left(u_{1}\right)\right]_{\equiv_{L}}, \ldots,\left[\pi_{\Sigma_{1}}\left(u_{k}\right)\right]_{\equiv_{L}}\right) \in F_{1} \times \ldots \times F_{k} .
$$

Choose $u \in \prod_{i=1}^{k} \pi_{\Sigma_{i}}\left(u_{i}\right)$. By assumption, $\prod_{i=1}^{k} \pi_{\Sigma_{i}}(L) \subseteq L$. Hence $u \in L$. As $\pi_{\Sigma_{i}}(u)=\pi_{\Sigma_{i}}\left(u_{i}\right)$ for $i \in\{1, \ldots, k\}$, we find

$$
\left(\left[\pi_{\Sigma_{1}}\left(u_{1}\right)\right]_{\equiv_{L}}, \ldots,\left[\pi_{\Sigma_{1}}\left(u_{k}\right)\right]_{\equiv_{L}}\right)=\left(\left[\pi_{\Sigma_{1}}(u)\right]_{\equiv_{L}}, \ldots,\left[\pi_{\Sigma_{1}}(u)\right]_{\equiv_{L}}\right) .
$$

By Definition 13. $\left(\left[\pi_{\Sigma_{1}}(u)\right]_{\equiv_{L}}, \ldots,\left[\pi_{\Sigma_{1}}(u)\right]_{\equiv_{L}}\right) \in F$. So, $F_{1} \times \ldots \times F_{k} \subseteq F$. 
2. Assume $F=F_{1} \times \ldots \times F_{k}$.

Let $u \in \bigsqcup_{i=1}^{k} \pi_{\Sigma_{i}}(L)$. Then there exist words $u_{i} \in L, i \in\{1, \ldots, k\}$, such that $\pi_{\Sigma_{i}}(u)=\pi_{\Sigma_{i}}\left(u_{i}\right)$. So, $\left[\pi_{\Sigma_{i}}\left(u_{i}\right)\right]_{\equiv_{L}} \in F_{i}$ and we find

$$
\left(\left[\pi_{\Sigma_{1}}(u)\right]_{\equiv_{L}}, \ldots,\left[\pi_{\Sigma_{k}}(u)\right]_{\equiv_{L}}\right) \in F .
$$

By Definition 13, there exists $v \in L$ such that

$$
\left(\left[\pi_{\Sigma_{1}}(u)\right]_{\equiv_{L}}, \ldots,\left[\pi_{\Sigma_{k}}(u)\right]_{\equiv_{L}}\right)=\left(\left[\pi_{\Sigma_{1}}(v)\right]_{\equiv_{L}}, \ldots,\left[\pi_{\Sigma_{k}}(v)\right]_{\equiv_{L}}\right) .
$$

Using Remark 6 and the previous equation,

$$
\begin{aligned}
{[u]_{\equiv_{L}} } & =\left[\pi_{\Sigma_{1}}(u) \cdots \pi_{\Sigma_{k}}(u)\right]_{\equiv_{L}} \\
& =\left[\pi_{\Sigma_{1}}(u)\right]_{\equiv_{L}} \cdot \ldots \cdot\left[\pi_{\Sigma_{k}}(u)\right]_{\equiv L} \\
& =\left[\pi_{\Sigma_{1}}(v)\right]_{\equiv_{L}} \cdot \ldots \cdot\left[\pi_{\Sigma_{k}}(v)\right]_{\equiv L} \\
& =[v]_{\equiv L}
\end{aligned}
$$

So, as $v \in L$, we have $u \in L$. Hence, $\prod_{i=1}^{k} \pi_{\Sigma_{i}}(L) \subseteq L$. So, with Equation (7), $L=\prod_{i=1}^{k} \pi_{\Sigma_{i}}(L)$.

Hence, the first and the last condition are equivalent and this concludes the proof.

Theorem 21, We have $\mathcal{L}_{1} \subsetneq \mathcal{L}_{2} \subsetneq \mathcal{L}_{3}$.

Proof. By Proposition 28, we find $\mathcal{L}_{1} \subseteq \mathcal{L}_{2}$. That the inclusion is proper is shown by Example 10. Suppose $L=\bigsqcup_{i=1}^{k} \pi_{\Sigma_{i}}(L)$. Let $i \in\{1, \ldots, k\}$ and $u, v \in \Sigma_{i}^{*}$. We have

$$
u \equiv_{L} v \Leftrightarrow \forall x \in \Sigma^{*}: u x \in L \leftrightarrow v x \in L .
$$

Using Proposition 25, the condition on the right hand side is equivalent to

$$
\begin{aligned}
\forall x \in \Sigma^{*}\left[\left(\forall j \in\{1, \ldots, k\}: \pi_{\Sigma_{j}}(u x)\right.\right. & \left.\in \pi_{\Sigma_{j}}(L)\right) \leftrightarrow \\
& \left.\left(\forall j \in\{1, \ldots, k\}: \pi_{\Sigma_{j}}(v x) \in \pi_{\Sigma_{j}}(L)\right)\right] .
\end{aligned}
$$

Claim: Equation (9) is equivalent to

$$
\forall x \in \Sigma^{*}: \pi_{\Sigma_{i}}(u x) \in \pi_{\Sigma_{i}}(L) \leftrightarrow \pi_{\Sigma_{i}}(v x) \in \pi_{\Sigma_{i}}(L) .
$$

Proof of the Claim: Suppose Equation (10) holds true. Let $x \in \Sigma^{*}$. Then, if, for each $j \in\{1, \ldots, k\}$, we have

$$
\pi_{\Sigma_{j}}(u x) \in \pi_{\Sigma_{j}}(L),
$$

using that, for $u, v \in \Sigma_{i}^{*}$, we have,

$$
\pi_{\Sigma_{j}}(u x)=\left\{\begin{array}{l}
u \pi_{\Sigma_{i}}(x) \text { if } i=j \\
\pi_{\Sigma_{j}}(x) \text { if } i \neq j
\end{array}\right.
$$


and similarly for $\pi_{\Sigma_{j}}(v x)$, we find with Equation (10) that, for each $j \in\{1, \ldots, k\}$,

$$
\left.\pi_{\Sigma_{j}}(u x) \in \pi_{\Sigma_{j}}(L)\right) .
$$

The other implication of Equation (9) can be shown similarly. Hence, Equation (9) holds true.

Conversely, suppose now Equation (9) holds true. Let $x \in \Sigma^{*}$ and assume

$$
\pi_{\Sigma_{i}}(u x) \in \pi_{\Sigma_{i}}(L) .
$$

As $L \neq \varnothing$, we find $u_{j} \in \Sigma_{j}^{*}$ such that $u_{j} \in \pi_{\Sigma_{j}}(L)$ for each $j \in\{1, \ldots, k\}$. Then, set $y=u_{1} \cdots u_{i-1} \pi_{\Sigma_{i}}(x) u_{i+1} \cdots u_{k}$. By choice and as $\pi_{\Sigma_{i}}(y)=$ $\pi_{\Sigma_{i}}(x)$, we have for each $j \in\{1, \ldots, k\}$ that

$$
\pi_{\Sigma_{j}}(u y) \in \pi_{j}(L)
$$

Hence, by Equation (9), for each $j \in\{1, \ldots, k\}$,

$$
\pi_{\Sigma_{j}}(v y) \in \pi_{j}(L) .
$$

In particular, $\pi_{\Sigma_{i}}(v x)=\pi_{\Sigma_{i}}(v y) \in \pi_{i}(L)$. The other implication of Equation (10) can be shown similarly. Hence, Equation (10) holds true. [End, Proof of the Claim]

So, by the previous claim, Equation (9) simplifies to

$$
\forall x \in \Sigma^{*}: \pi_{\Sigma_{i}}(u x) \in \pi_{\Sigma_{i}}(L) \leftrightarrow \pi_{\Sigma_{i}}(v x) \in \pi_{\Sigma_{i}}(L),
$$

which is equivalent to

$$
\forall x \in \Sigma_{i}^{*}: u x \in \pi_{\Sigma_{i}}(L) \leftrightarrow v x \in \pi_{\Sigma_{i}}(L)
$$

But this is precisely the definition of $u \equiv_{\pi_{\Sigma_{i}}(L)} v$. Hence,

$$
[u]_{\equiv_{L}} \cap \Sigma_{i}^{*}=[u]_{\pi_{\Sigma_{i}}(L)} \cap \Sigma_{i}^{*}
$$

and by Proposition 26 , we find $\mathcal{L}_{2} \subseteq \mathcal{L}_{3}$. That the inclusion is proper, is shown by Example 4 .

\section{References for the Appendix}

Hop71. John Hopcroft. An $n \log n$ algorithm for minimizing states in a finite automaton. In Theory of machines and computations (Proc. Internat. Sympos., Technion, Haifa, 1971), pages 189-196. Academic Press, New York, 1971.

Koz97. Dexter Kozen. Automata and computability. Undergraduate texts in computer science. Springer, 1997.

YZS94. S. Yu, Q. Zhuang, and K. Salomaa. The state complexities of some basic operations on regular languages. Theor. Comput. Sci., 125(2):315-328, March 1994. 